\begin{tabular}{|c|c|}
\hline Title & Timelike minimal Lagrangian surfaces in the indefinite complex hy perbolic two-space \\
\hline Author(s) & Dorfmesiter, Josef F.; Kobayashi, Shimpei \\
\hline Citation & $\begin{array}{l}\text { A nnali di matematica pura ed applicata, 200(2), 521-546 } \\
\text { https://doi.org/10.1007/s10231-020-01005-1 }\end{array}$ \\
\hline Issue Date & $2020-06-05$ \\
\hline Doc URL & http:/hdl.handle.net/2115/81689 \\
\hline Rights & $\begin{array}{l}\text { This is a post-peer-review, pre copy edit version of an article published in A nnali di Matematica Pura ed A pplicata. The } \\
\text { final authenticated version is available online at: } \mathrm{http} / / / \mathrm{dx} \text {.doi.org/10.1007/s10231-020-01005-1 }\end{array}$ \\
\hline Tyре & article (author version) \\
\hline File Information & A nn. Mat. Pura A ppl. 200-2_521-546.pdf \\
\hline
\end{tabular}

Instructions for use 


\title{
TIMELIKE MINIMAL LAGRANGIAN SURFACES IN THE INDEFINITE COMPLEX HYPERBOLIC TWO-SPACE
}

\author{
JOSEF F. DORFMEISTER AND SHIMPEI KOBAYASHI
}

\begin{abstract}
It has been known for some time that there exist 5 essentially different real forms of the complex affine Kac-Moody algebra of type $A_{2}^{(2)}$ and that one can associate 4 of these real forms with certain classes of "integrable surfaces", such as minimal Lagrangian surfaces in $\mathbb{C P}^{2}$ and $\mathbb{C H}^{2}$, as well as definite and indefinite affine spheres in $\mathbb{R}^{3}$.

In this paper we consider the class of timelike minimal Lagrangian surfaces in the indefinite complex hyperbolic two-space $\mathbb{C H}_{1}^{2}$. We show that this class of surfaces corresponds to the fifth real form.

Moreover, for each timelike Lagrangian surface in $\mathbb{C H}_{1}^{2}$ we define natural Gauss maps into certain homogeneous spaces and prove a Ruh-Vilms type theorem, characterizing timelike minimal Lagrangian surfaces among all timelike Lagrangian surfaces in terms of the harmonicity of these Gauss maps.
\end{abstract}

\section{INTRODUCTION}

It became more and more clear in recent years that many surface classes are characterized by harmonic maps into some $k$-symmetric space. In the classical case Ruh-Vilms [25] have characterized all constant mean curvature surfaces in $\mathbb{R}^{3}$ among all surfaces as those for which the (classical) Gauss map into the symmetric space $\mathbb{S}^{2}=\mathrm{SO}_{3} / \mathrm{SO}_{2}$ is harmonic. Another case consists of all constant mean curvature surfaces in the real hyperbolic space $\mathbb{H}^{3}$, which are those surfaces in $\mathbb{H}^{3}$ for which the "normal Gauss map" into the unit tangent bundle of $\mathbb{H}^{3}$, considered as a 4-symmetric space, is harmonic, [9]. Another group of surfaces with an analogous characterization seem to be the definite and the indefinite affine spheres, and the minimal Lagrangian surfaces in $\mathbb{C P}^{2}$ and in $\mathbb{C H}^{2}$, see for examples, $[7,12,11,21]$. (So far only in [23] a harmonic "Gauss map" is given explicitly.)

All these examples come in $S^{1}$-families of surfaces of the same class and can be investigated by using the loop group technique. Here one observes that the naturally associated moving frames of an associated family are contained in a specific loop group. In [7] it was observed that the indefinite affine spheres in $\mathbb{R}^{3}$ are associated with a real form of the affine KacMoody algebra of type $A_{2}^{(2)}$. Later it was observed that the definite affine spheres (of elliptic type or of hyperbolic type) also are associated with a real form of type $A_{2}^{(2)}$, as well as

Date: May 3, 2020.

2010 Mathematics Subject Classification. Primary 53A10, 53B30, 58D10, Secondary 53C42.

Key words and phrases. Timelike minimal Lagrangian surfaces; Loop groups; Real forms; Tzitzéica equations.

The second named author is partially supported by JSPS KAKENHI Grant Number JP18K03265 and Deutsche Forschungsgemeinschaft-Collaborative Research Center, TRR 109, "Discretization in Geometry and Dynamics". 
the minimal Lagrangian immersions into $\mathbb{C P}^{2}$ and the the minimal Lagrangian immersions into $\mathbb{C H}^{2}$. In view of the classification of all real forms of the complex affine Kac-Moody algebra of type $A_{2}^{(2)}$ by Heintze-Groß [13] or Rousseau et al. [3, 4] it became clear, that the surface types mentioned above correspond exactly to four of the five types of inequivalent real forms, see [8]. For the case of the complex affine Kac-Moody algebra of type $A_{1}^{(1)}$ it has been shown in [18] that the real forms of this Kac-Moody algebra are related to constant mean curvature/constant Gaussian curvature surfaces in the Euclidean 3-space, the Minkowski 3 -space or in the hyperbolic 3-space.

In this paper we present the "missing case". More precisely, we define timelike minimal Lagrangian surfaces in the indefinite complex hyperbolic space which are associated with the missing real form, and also define a Gauss map for all Lagrangian surfaces in the indefinite complex hyperbolic space. These Gauss maps take values in a quasi 6-symmetric space (see Definition 5) and are Lorentz primitive harmonic if and only if the corresponding Lagrangian surfaces in the indefinite complex hyperbolic space are minimal. We note that in $[10,14]$, the loop group methods for timelike constant mean curvature surfaces and timelike minimal surfaces in the Minkowski 3-space $\mathbb{R}_{1}^{3}$ have been developed, and these surfaces correspond to a real form of the complex affine Kac-Moody algebra of type $A_{1}^{(1)}$.

This result permits to apply the loop group technique which represents a general procedure to construct all surfaces of the associated class; in our case all minimal timelike Lagrangian surfaces in the indefinite complex hyperbolic space. More on this is left to a separate investigation.

\section{Timelike minimal Lagrangian surfaces in $\mathbb{C H}_{1}^{2}$}

In this section, we define timelike Lagrangian surfaces in $\mathbb{C H}_{1}^{2}$ and discuss their basic properties. In particular we characterize minimality of a timelike Lagrangian surface by the vanishing of the so-called "mean curvature" 1-form, Proposition 1.11.

\subsection{Surfaces in $\mathbb{C H}_{1}^{2}$. Let}

$$
P_{0}=\left(\begin{array}{ccc}
0 & 1 & 0 \\
1 & 0 & 0 \\
0 & 0 & -1
\end{array}\right)
$$

and consider the three-dimensional complex Hermitian flat space $\mathbb{C}_{2}^{3}$, that is, $\mathbb{C}^{3}$ together with the pseudo-Hermitian form of signature $(2,1)$

$$
\langle z, w\rangle=z^{T} P_{0} \bar{w}=z_{1} \overline{w_{2}}+z_{2} \overline{w_{1}}-z_{3} \overline{w_{3}} .
$$

Vectors $v \in \mathbb{C}_{2}^{3}$ satisfying $\langle v, v\rangle<0$ or $\langle v, v\rangle>0$ will be called "negative" and "positive" respectively. Clearly, the set of these vectors is open in $\mathbb{C}_{2}^{3}$ and $\mathbb{C}^{\times}$acts freely on these sets by multiplication.

Definition 1. The real part and the imaginary part of the indefinite Hermitian inner product of $\mathbb{C}_{2}^{3}$ define a pseudo-Riemannian metric $g$ and a symplectic form $\Omega$, respectively:

$$
\langle,\rangle=\operatorname{Re}\langle,\rangle+\sqrt{-1} \operatorname{Im}\langle,\rangle=g(,)+\sqrt{-1} \Omega(,) .
$$


Then the indefinite complex hyperbolic space $\mathbb{C H}_{1}^{2}$, see [6, Section 2], defined by

$$
\mathbb{C H}_{1}^{2}=\left\{\mathbb{C}^{\times} v \mid v \in \mathbb{C}_{2}^{3},\langle v, v\rangle<0\right\}
$$

is a two-dimensional complex manifold. Let

$$
\mathrm{U}_{2,1}=\left\{\begin{array}{l|l}
A \mid \begin{array}{l}
\text { Invertible real linear map in } \mathbb{C}_{2}^{3} \\
\text { satisfying }\langle A v, A w\rangle=\langle v, w\rangle \text { for all } v, w \in \mathbb{C}_{2}^{3} .
\end{array}
\end{array}\right\} .
$$

Then $\mathbb{C}^{\times} \cdot \mathrm{U}_{2,1}$ is a connected reductive Lie group which acts transitively on the set of negative (resp. positive) vectors. As a consequence, $\mathrm{U}_{2,1}$ acts transitively on $\mathbb{C H}_{1}^{2}$ and it is easy to verify that the stabilizer in $\mathrm{U}_{2,1}$ of the negative vector $e_{3}=(0,0,1)^{T}$ is given by the diagonal block form matrix group $\mathrm{U}_{1,1} \times \mathrm{U}_{1}$ in $\mathrm{U}_{2,1}$, where $\mathrm{U}_{1,1}$, is the group of isometries of the indefinite Hermitian metric of $\mathbb{C}_{1}^{2}$ given by $(z, w)=z_{1} \overline{w_{2}}+z_{2} \overline{w_{1}}$.

As a consequence, $\mathbb{C H}_{1}^{2}$ can be represented as the indefinite Hermitian symmetric space, see for example [26, Section 2]:

$$
\mathbb{C H}_{1}^{2}=\mathrm{U}_{2,1} / \mathrm{U}_{1,1} \times \mathrm{U}_{1} .
$$

The complex manifold $\mathbb{C H}_{1}^{2}$ carries naturally the pseudo-Hermitian metric induced from $\mathbb{C}_{2}^{3}$. The projection is a pseudo-Riemannian submersion.

Remark 1.1. The indefinite complex hyperbolic space $\mathbb{C H}_{1}^{2}$ is known to be anti-isometric (the metrics differ by a minus sign) to the complex de Sitter space $\mathbb{C P}_{1}^{2}$ of all positive lines of complex Hermitian flat space $\mathbb{C}_{1}^{3}$ with signature $(1,2)$, see $[1$, p. 96].

Let $H_{3}^{5}$ be the anti-de Sitter sphere (note again that the signature of $\mathbb{C}_{2}^{3}$ is $(2,1)$ ):

$$
H_{3}^{5}=\left\{v \in \mathbb{C}_{2}^{3} \mid\langle v, v\rangle=-1\right\} .
$$

Then there exists the Boothby-Wang type fibration $\pi: H_{3}^{5} \rightarrow \mathbb{C H}_{1}^{2}$ given by $v \mapsto \mathbb{C}^{\times} v,[5,6]$. The tangent space of $H_{3}^{5}$ at $p \in H_{3}^{5}$ is

$$
T_{p} H_{3}^{5}=\left\{w \in \mathbb{C}_{2}^{3} \mid \operatorname{Re}\langle w, p\rangle=0\right\} .
$$

Moreover, the space

$$
\mathcal{H}_{p}=\left\{w \in T_{p} H_{3}^{5} \mid\langle w, p\rangle=0\right\}
$$

is a natural horizontal subspace. Recall that the projection $\pi$ from $H_{3}^{5}$ to $\mathbb{C H}_{1}^{2}$ is a pseudoRiemannian submersion. Moreover, note that the form

$$
\zeta(p)=\operatorname{Im}\langle p, \cdot\rangle
$$

is a contact form and $H_{3}^{5}$ is a contact manifold. Note also that $H_{3}^{5}$ can be represented as the symmetric space

$$
H_{3}^{5}=\mathrm{U}_{2,1} / \mathrm{U}_{1,1}
$$

where $U_{1,1}$ here more precisely means the block form matrix group $U_{1,1} \times\{1\}$.

Since $\pi$ is a pseudo-Riemannian submersion, we will make use of the pseudo-Riemannian metric $g$ and the symplectic form $\Omega$ on $\mathbb{C H}_{1}^{2}$ which is given by

$$
g(a, b)=\operatorname{Re}\langle\tilde{a}, \tilde{b}\rangle, \quad \Omega(a, b)=\operatorname{Im}\langle\tilde{a}, \tilde{b}\rangle,
$$

where $a, b \in T_{p} \mathbb{C} \mathbb{H}_{1}^{2}$ and $\tilde{a}, \tilde{b} \in T_{\tilde{p}} H_{3}^{5}$ are the vectors in the horizontal subspace $\mathcal{H}_{\tilde{p}} \subset T_{\tilde{p}} H_{3}^{5}$ corresponding uniquely to $a$ and $b$ respectively via $\pi$. 
Lemma 1.2. Let $\mathbb{D}$ be a simply connected domain in $\mathbb{R}^{2}$ and $f: \mathbb{D} \rightarrow \mathbb{C H}_{1}^{2}$ a Lagrangian map, (thus satisfying $\Omega(\mathrm{d} f, \mathrm{~d} f)=0$ ). Then there exists a lift $\mathfrak{f}: \mathbb{D} \rightarrow H_{3}^{5}$ such that

$$
\langle\mathrm{d} \mathfrak{f}, \mathfrak{f}\rangle=0 .
$$

This lift is unique up to a constant factor from $S^{1}$. A lift $\mathfrak{f}$ of a Lagrangian map $f$ with the condition (1.7) as above will be called a horizontal lift.

Proof. Let $\hat{\mathfrak{f}}: \mathbb{D} \rightarrow H_{3}^{5}$ be a lift of $f$. Then $\langle\mathrm{d} \hat{\mathfrak{f}}, \hat{\mathfrak{f}}\rangle+\langle\hat{\mathfrak{f}}, \mathrm{d} \hat{\mathfrak{f}}\rangle=0$, that is, $\langle\mathrm{d} \hat{\mathfrak{f}}, \hat{\mathfrak{f}}\rangle$ takes purely imaginary values. Moreover, the Lagrangian condition for $\mathfrak{f}$ means that $\langle\mathrm{d} \hat{\mathfrak{f}}, \hat{\mathfrak{f}}\rangle$ is a closed 1 -form. Since $\mathbb{D}$ is a simply connected domain in $\mathbb{R}^{2}$, the form $\langle\mathrm{d} \hat{\mathfrak{f}}, \hat{\mathfrak{f}}\rangle$ is exact. Hence there exists a real function $\eta: \mathbb{D} \rightarrow \mathbb{R}$ such that $\sqrt{-1} \mathrm{~d} \eta=\langle\mathrm{d} \hat{\mathfrak{f}}, \hat{\mathfrak{f}}\rangle$. Then we put $\mathfrak{f}=e^{\sqrt{-1} \eta \hat{\mathfrak{f}}}$ and $\langle\mathrm{d} \mathfrak{f}, \mathfrak{f}\rangle=0$ follows.

Remark 1.3. A horizontal lift $\mathfrak{f}: \mathbb{D} \rightarrow H_{3}^{5}$ of $f$ is sometimes called a Legendre lift of $f: \mathbb{D} \rightarrow$ $\mathbb{C H}_{1}^{2}$, since for a horizontal lift of a Lagrangian immersion the condition (1.7) is equivalent with $\zeta(\mathfrak{f}(q))(\mathrm{d} \mathfrak{f}(q))=0$, equivalently $\operatorname{Im}\langle\mathrm{d} \mathfrak{f}, \mathfrak{f}\rangle=0$, and this means that $\mathfrak{f}$ is a Legendre immersion into the contact manifold $H_{3}^{5}$. For a more general discussion of the notion a Legendre lift see Section 3.

Let $f: M \rightarrow \mathbb{C H}_{1}^{2}$ be a Lagrangian immersion from a two-dimensional manifold $M$. Then $f$ induces a pseudo-Riemannian metric on $M$. If we restrict the immersion $f$ to any contractible open subset $\mathbb{D}$ of $M$, then the induced metric of $f$ is represented, on $\mathbb{D}$, by using the horizontal lift $\mathfrak{f}$, as

$$
\mathrm{d} s^{2}=\operatorname{Re}\langle\mathrm{d} \mathfrak{f}, \mathrm{d} \mathfrak{f}\rangle=g(\mathrm{~d} f, \mathrm{~d} f) .
$$

Note, the second equality above comes from the fact that two horizontal lifts of $f$ only differ by a constant scalar factor from $S^{1}$.

In what follows we will consider exclusively timelike surfaces. Hence the induced metric $\mathrm{d} s^{2}$ is assumed to be indefinite. Moreover, we always assume that all surfaces are Lagrangian.

Remark 1.4. For a Lagrangian immersion $f$ in $\mathbb{C H}_{1}^{2}$, we obtain for the complex structure $J$ of $\mathbb{C H}_{1}^{2}$ the identity $g(J \circ \mathrm{d} f, J \circ \mathrm{d} f)=g(\mathrm{~d} f, \mathrm{~d} f)$. The definition of a Lagrangian surface implies that $J \circ \mathrm{d} f$ is perpendicular to $\mathrm{d} f$ and a timelike vector. As a consequence, $g(\mathrm{~d} f, \mathrm{~d} f)$ is not spacelike. Hence we have the following corollary.

Corollary 1.5. There does not exist any spacelike Lagrangian surface in $\mathbb{C H}_{1}^{2}$.

1.2. Moving frame. In this subsection we discuss moving frames of timelike Lagrangian surfaces in $\mathbb{C H}_{1}^{2}$. First we discuss null coordinates, that is, for an indefinite metric $g=$ $\sum_{i, j} g_{i j} \mathrm{~d} x_{i} \mathrm{~d} x_{j}$ on a surface $M$, such that $g_{11}=g_{22}=0$ and $g_{12}=g_{21} \neq 0$. The existence of null coordinates can be found for example in [27] or [2, Prop 14.1.18 and Remark 14.1.19]. In our case, this result is formulated as follows:

Theorem 1.6. Let $f: \mathbb{D} \rightarrow \mathbb{C H}_{1}^{2}$ be a timelike Lagrangian immersion and $\mathfrak{f}$ a horizontal lift of $\mathfrak{f}$. Then the metric (1.8) induced by $\mathfrak{f}($ and $f)$ on $\mathbb{D}$ is Lorentzian. In particular, in a neighbourhood of any point of $\mathbb{D}$ null coordinates exist for $\mathfrak{f}$ (and $f$ ). 
For a horizontal lift $\mathfrak{f}$ of a timelike Lagrangian immersion $f$ we thus have:

$$
\operatorname{Re}\left\langle\mathfrak{f}_{u}, \mathfrak{f}_{u}\right\rangle=\operatorname{Re}\left\langle\mathfrak{f}_{v}, \mathfrak{f}_{v}\right\rangle=0, \quad \text { and } \operatorname{Re}\left\langle\mathfrak{f}_{u}, \mathfrak{f}_{v}\right\rangle \text { never vanishes. }
$$

From the horizontality $\langle\mathrm{d} \mathfrak{f}, \mathfrak{f}\rangle=0$, we have $\left\langle\mathfrak{f}_{u}, \mathfrak{f}\right\rangle=\left\langle\mathfrak{f}_{v}, \mathfrak{f}\right\rangle=0$. Moreover, taking the derivative with respect to $v$ and $u$, respectively, we obtain $\left\langle\mathfrak{f}_{u}, \mathfrak{f}_{v}\right\rangle=\left\langle\mathfrak{f}_{v}, \mathfrak{f}_{u}\right\rangle$. Hence, $\left\langle\mathfrak{f}_{u}, \mathfrak{f}_{v}\right\rangle$ is real and never vanishes. Assuming without loss of generality $\left\langle\mathfrak{f}_{v}, \mathfrak{f}_{u}\right\rangle>0$ we finally obtain:

$$
\left\langle\mathfrak{f}_{u}, \mathfrak{f}_{u}\right\rangle=\left\langle\mathfrak{f}_{v}, \mathfrak{f}_{v}\right\rangle=0, \quad \text { and }\left\langle\mathfrak{f}_{u}, \mathfrak{f}_{v}\right\rangle=\left\langle\mathfrak{f}_{v}, \mathfrak{f}_{u}\right\rangle \text { is always positive, }
$$

and

$$
\operatorname{Im}\left\langle\mathfrak{f}_{u}, \mathfrak{f}_{v}\right\rangle=\Omega\left(f_{u}, f_{v}\right)=0 .
$$

Moreover, we have just seen that $\left\langle\mathfrak{f}_{u}, \mathfrak{f}_{v}\right\rangle$ is always positive. Therefore, we can assume that there exists a real function $\omega: \mathbb{D} \rightarrow \mathbb{R}$ such that

$$
\left\langle\mathfrak{f}_{u}, \mathfrak{f}_{v}\right\rangle=e^{\omega} \quad \text { and } \quad \mathrm{d} s^{2}=2 e^{\omega} \mathrm{d} u \mathrm{~d} v
$$

holds. Then we consider the coordinate frame

$$
\mathcal{F}=\left(e^{-\omega / 2} \mathfrak{f}_{u}, e^{-\omega / 2} \mathfrak{f}_{v}, \mathfrak{f}\right) .
$$

It is straightforward to see that $\mathcal{F}$ takes values in $\mathrm{U}_{2,1}$, that is,

$$
P_{0} \mathcal{F}^{T} P_{0} \overline{\mathcal{F}}=\mathrm{id}, \quad \text { equivalently } \quad \mathcal{F}^{-1}=P_{0} \overline{\mathcal{F}}^{T} P_{0}
$$

holds, where $P_{0}$ is defined in (1.1). Then $|\operatorname{det} \mathcal{F}|^{2}=1$ and $\mathcal{F} \in \mathrm{U}_{2,1}=S^{1} \cdot \mathrm{SU}_{2,1}$ follows.

We now want to compute the Maurer-Cartan form of $\mathcal{F}$. For this we will use the mean curvature vector of $f$.

First we consider the decomposition

$$
\mathbb{D} \times \mathbb{C}_{2}^{3}=\mathrm{d} \mathfrak{f}\left(\mathbb{R}^{2}\right) \oplus \mathrm{d} \mathfrak{f}\left(\mathbb{R}^{2}\right)^{\perp} \oplus \mathrm{d} \mathfrak{f}\left(\mathbb{R}^{2}\right)^{\perp \perp}
$$

of the trivial bundle into three real, pairwise perpendicular rank 2 subbundles, where

$$
\mathcal{H}=\mathrm{d} \mathfrak{f}\left(\mathbb{R}^{2}\right) \oplus \mathrm{d} \mathfrak{f}\left(\mathbb{R}^{2}\right)^{\perp}
$$

is the natural horizontal subspace of $T_{\mathfrak{f}(z)} H_{3}^{5}$. The vectors $\left\{\mathfrak{f}_{u}, \mathfrak{f}_{v}\right\},\left\{\sqrt{-1} \mathfrak{f}_{u}, \sqrt{-1} \mathfrak{f}_{v}\right\}$, and $\{\sqrt{-1} \mathfrak{f}, \mathfrak{f}\}$ form a basis of these real two-dimensional subspaces respectively. Their pairwise Hermitian products can be read off from the formulas listed just above. By the definition of the metric $g$ the projection $\hat{\pi}$ from $\mathbb{D} \times \mathbb{C}_{2}^{3}$ to $\mathbb{C}_{2}^{3}$ induces an isometry from $\mathrm{d} \mathfrak{f}\left(\mathbb{R}^{2}\right)$ onto $\mathrm{d} f\left(\mathbb{R}^{2}\right)$ and from $\mathrm{d} \mathfrak{f}\left(\mathbb{R}^{2}\right)^{\perp}$ onto $\mathrm{d} f\left(\mathbb{R}^{2}\right)^{\perp}$. The vector $\sqrt{-1} \mathfrak{f}$ is annihilated by $\mathrm{d} \pi$.

More precisely, putting $E_{1}=\mathfrak{f}_{u}$ and $E_{2}=\mathfrak{f}_{v}$ we obtain basis vectors of $\mathrm{d} \mathfrak{f}\left(\mathbb{R}^{2}\right)$. Then $\sqrt{-1} E_{1}=\sqrt{-1} \mathfrak{f}_{u}$ and $\sqrt{-1} E_{2}=\sqrt{-1} \mathfrak{f}_{v}$ and $\sqrt{-1} \mathfrak{f}_{u}$ and $\sqrt{-1} \mathfrak{f}_{v}$ are in $\mathrm{d} \mathfrak{f}\left(\mathbb{R}^{2}\right)^{\perp}$. The differential of $\pi$ maps the vectors onto $\mathrm{d} f\left(\mathbb{R}^{2}\right)$ and $\mathrm{d} f\left(\mathbb{R}^{2}\right)^{\perp}$, respectively.

Recall that from [24, Theorem 1, c)], the second fundamental form $I I^{f}$ for a timelike Lagrangian surface $f$ in $\mathbb{C H}_{1}^{2}$ can be obtained by the second fundamental form $I I^{\mathfrak{f}}$ for a horizontal lift $\mathfrak{f}$ in $H_{3}^{5}$ which takes values in the horizontal subspace $\mathcal{H}$, given as $I I^{f}=\mathrm{d} \pi I I^{\mathfrak{f}}$ : see [24, Theorem 1]. Since $\mathfrak{f}$ is in $H_{3}^{5} \subset \mathbb{C}_{2}^{3}$, and $X Y \mathfrak{f}$ takes values in $\mathbb{C}_{2}^{3}$ and the second fundamental form $I I^{\mathfrak{f}}$ can be given by

$$
I I^{\mathfrak{f}}(X, Y)=g\left(X Y \mathfrak{f}, e_{1}\right) e_{1}-g\left(X Y \mathfrak{f}, e_{2}\right) e_{2},
$$


with $X, Y \in \Gamma(T \mathbb{D})$ and $e_{1}$ and $e_{2}$ being perpendicular vectors of $\mathrm{d} \mathfrak{f}\left(\mathbb{R}^{2}\right)^{\perp}$ of "length" 1 and -1 respectively. Note that $I I^{\mathfrak{f}}(X, Y)$ takes values in $T_{p} \mathcal{H}$. Then the mean curvature vector $\mathfrak{H}$ of $\mathfrak{f}$ is defined by $\frac{1}{2} \operatorname{Tr}_{g} I I^{\mathfrak{f}}$, that is,

$$
\mathfrak{H}=\frac{1}{2}\left\{I I^{\mathfrak{f}}\left(\partial_{s}, \partial_{s}\right)-I I^{\mathfrak{f}}\left(\partial_{t}, \partial_{t}\right)\right\}=\frac{1}{2}\left\{g\left(\partial_{s}^{2} \mathfrak{f}-\partial_{t}^{2} \mathfrak{f}, e_{1}\right) e_{1}-g\left(\partial_{s}^{2} \mathfrak{f}-\partial_{t}^{2} \mathfrak{f}, e_{2}\right) e_{2}\right\},
$$

where $\left\{\partial_{s}, \partial_{t}\right\}$ is the orthonormal frame with respect to the indefinite metric $\mathrm{d} s^{2}$. In our case we define $e_{1}=\frac{\sqrt{-1}}{\sqrt{2}}\left(E_{1}+E_{2}\right) e^{-\frac{\omega}{2}}$ and $e_{2}=\frac{\sqrt{-1}}{\sqrt{2}}\left(E_{1}-E_{2}\right) e^{-\frac{\omega}{2}}$ and $\partial_{s}=\frac{1}{\sqrt{2}} e^{-\frac{\omega}{2}}\left(\partial_{u}+\partial_{v}\right)$, $\partial_{t}=\frac{1}{\sqrt{2}} e^{-\frac{\omega}{2}}\left(\partial_{u}-\partial_{v}\right)$. Then, from equation (1.13) it follows by a straightforward computation

$$
\mathfrak{H}=e^{-2 \omega}\left\{g\left(\mathfrak{f}_{u v}, \sqrt{-1} E_{1}\right) \sqrt{-1} E_{2}+g\left(\mathfrak{f}_{u v}, \sqrt{-1} E_{2}\right) \sqrt{-1} E_{1}\right\} .
$$

Now the mean curvature $H$ of the original immersion $f$ is given by

$$
\mathrm{d} \pi(\mathfrak{H})=H .
$$

By abuse of notation we will also call $\mathfrak{H}$ the mean curvature vector of $f$. A straightforward computation shows that we obtain the following description of $\mathfrak{H}$ :

$$
\mathfrak{H}=g\left(e^{-\omega} \mathfrak{f}_{u v}, \mathfrak{E}_{1}\right) \mathfrak{E}_{2}+g\left(e^{-\omega} \mathfrak{f}_{u v}, \mathfrak{E}_{2}\right) \mathfrak{E}_{1},
$$

where $\left\{\mathfrak{E}_{1}, \mathfrak{E}_{2}\right\}=\left\{\sqrt{-1} e^{-\omega / 2} \mathfrak{f}_{u}, \sqrt{-1} e^{-\omega / 2} \mathfrak{f}_{v}\right\}$ is a null basis of $\mathrm{d} \mathfrak{f}\left(\mathbb{R}^{2}\right)^{\perp}$. We thus compute $\mathfrak{H}$ as the component of $e^{-\omega} \mathfrak{f}_{u v}$ in $\mathrm{d} \mathfrak{f}\left(\mathbb{R}^{2}\right)^{\perp}$. Since $g\left(\mathfrak{f}_{u v}, \mathfrak{f}_{u}\right)=g\left(\mathfrak{f}_{u v}, \mathfrak{f}_{v}\right)=0$, we obtain $e^{-\omega} \mathfrak{f}_{u v}=$ $\mathfrak{H}+a i \mathfrak{f}+b \mathfrak{f}$. Taking inner products yields $b=-g\left(\mathfrak{f}_{u v}, \mathfrak{f}\right)=1$ and $a=0$. Thus altogether we obtain:

$$
\mathfrak{H}=e^{-\omega} \mathfrak{f}_{u v}-\mathfrak{f} .
$$

Remark 1.7. In general, for a surface $\mathfrak{f}$ in $H_{3}^{5}$, the second fundamental form can be written in the form

$$
I I(X, Y)=g\left(X Y \mathfrak{f}, \mathfrak{e}_{1}\right) \mathfrak{e}_{1}-g\left(X Y \mathfrak{f}, \mathfrak{e}_{2}\right) \mathfrak{e}_{2}-g\left(X Y \mathfrak{f}, \mathfrak{e}_{3}\right) \mathfrak{e}_{3},
$$

where $\left\{\mathfrak{e}_{1}, \mathfrak{e}_{2}, \mathfrak{e}_{3}\right\}$ are perpendicular vectors in $\mathrm{d} \mathfrak{f}\left(\mathbb{R}^{2}\right)^{\perp} \subset T_{\mathfrak{f}(p)} H_{3}^{5}$ of lengths 1 and -1 and -1 , respectively. If $\mathfrak{f}$ is a Legendre immersion, then $\mathfrak{e}_{3}=\sqrt{-1} \mathfrak{f}$ and $g\left(X Y \mathfrak{f}, \mathfrak{e}_{3}\right)=0$ from the Legendrian condition $\zeta(\mathfrak{f}(q))(\mathrm{d} \mathfrak{f}(q))=0$.

Now we can prove the following theorem.

Theorem 1.8. The Maurer-Cartan form $\mathcal{F}^{-1} \mathrm{~d} \mathcal{F}=\mathcal{F}^{-1} \mathcal{F}_{u} \mathrm{~d} u+\mathcal{F}^{-1} \mathcal{F}_{v} \mathrm{~d} v$ can be computed as

$$
\begin{aligned}
& \mathcal{U}=\mathcal{F}^{-1} \mathcal{F}_{u}=\left(\begin{array}{ccc}
\ell+\frac{\omega_{u}}{2} & m & e^{\omega / 2} \\
-Q e^{-\omega} & \ell-\frac{\omega_{u}}{2} & 0 \\
0 & e^{\omega / 2} & 0
\end{array}\right) \\
& \mathcal{V}=\mathcal{F}^{-1} \mathcal{F}_{v}=\left(\begin{array}{ccc}
m-\frac{\omega_{v}}{2} & -R e^{-\omega} & 0 \\
\ell & m+\frac{\omega_{v}}{2} & e^{\omega / 2} \\
e^{\omega / 2} & 0 & 0
\end{array}\right),
\end{aligned}
$$

where

$$
Q=\left\langle\mathfrak{f}_{\text {uuu }}, \mathfrak{f}\right\rangle, \quad R=\left\langle\mathfrak{f}_{\text {vvv }}, \mathfrak{f}\right\rangle, \quad \ell=\left\langle\mathfrak{H}, \mathfrak{f}_{u}\right\rangle, \quad m=\left\langle\mathfrak{H}, \mathfrak{f}_{v}\right\rangle,
$$

and $\mathfrak{H}$ is the mean curvature vector in (1.14). Moreover, $\ell, m, Q$ and $R$ take purely imaginary values. 
Proof. Writing $\mathcal{F}:=\left(\mathfrak{f}_{1}, \mathfrak{f}_{2}, \mathfrak{f}_{3}\right)$ and using the last equation in (1.12), we obtain $\mathcal{F}^{-1} \mathrm{~d} \mathcal{F}=$ $P_{0} \overline{\mathcal{F}}^{t} P_{0} \mathrm{~d} \mathcal{F}$. A straightforward computation now shows that

$$
\mathcal{U}=\mathcal{F}^{-1} \mathcal{F}_{u}=\left(\begin{array}{rrr}
\left\langle\mathfrak{f}_{1 u}, \mathfrak{f}_{2}\right\rangle & \left\langle\mathfrak{f}_{2 u}, \mathfrak{f}_{2}\right\rangle & \left\langle\mathfrak{f}_{3_{u}}, \mathfrak{f}_{2}\right\rangle \\
\left\langle\mathfrak{f}_{1_{u}}, \mathfrak{f}_{1}\right\rangle & \left\langle\mathfrak{f}_{2 u}, \mathfrak{f}_{1}\right\rangle & \left\langle\mathfrak{f}_{\mathfrak{f}_{u}}, \mathfrak{f}_{1}\right\rangle \\
-\left\langle\mathfrak{f}_{1_{u}}, \mathfrak{f}_{3}\right\rangle & -\left\langle\mathfrak{f}_{2_{u}}, \mathfrak{f}_{3}\right\rangle & -\left\langle\mathfrak{f}_{3_{u}}, \mathfrak{f}_{3}\right\rangle
\end{array}\right),
$$

and $\mathcal{V}$ is obtained from $\mathcal{U}$ by switching the subscripts $u$ and $v$. We want to compute the coefficients of $\mathcal{U}$ and $\mathcal{V}$ in more detail. First we note that by the definition of the coordinate frame $\mathcal{F}$ in (1.11), we have

$$
\mathfrak{f}_{1 u}=e^{-\omega / 2}\left(\mathfrak{f}_{u u}-\frac{\omega_{u}}{2} \mathfrak{f}_{u}\right), \quad \mathfrak{f}_{2 u}=e^{-\omega / 2}\left(\mathfrak{f}_{v u}-\frac{\omega_{u}}{2} \mathfrak{f}_{v}\right), \quad \mathfrak{f}_{3 u}=\mathfrak{f}_{u},
$$

A straightforward computation by using (1.18) shows that

$$
\begin{aligned}
\left\langle\mathfrak{f}_{1 u}, \mathfrak{f}_{2}\right\rangle & =e^{-\omega}\left\langle\mathfrak{f}_{u u}, \mathfrak{f}_{v}\right\rangle-\frac{\omega_{u}}{2}, & \left\langle\mathfrak{f}_{2 u}, \mathfrak{f}_{2}\right\rangle & =e^{-\omega}\left\langle\mathfrak{f}_{v u}, \mathfrak{f}_{v}\right\rangle, & \left\langle\mathfrak{f}_{3 u}, \mathfrak{f}_{2}\right\rangle & =e^{\omega / 2}, \\
\left\langle\mathfrak{f}_{1 u}, \mathfrak{f}_{1}\right\rangle & =e^{-\omega}\left\langle\mathfrak{f}_{u u}, \mathfrak{f}_{u}\right\rangle, & \left\langle\mathfrak{f}_{2 u}, \mathfrak{f}_{1}\right\rangle & =e^{-\omega}\left\langle\mathfrak{f}_{v u}, \mathfrak{f}_{u}\right\rangle-\frac{\omega_{u}}{2}, & \left\langle\mathfrak{f}_{3 u}, \mathfrak{f}_{1}\right\rangle & =0, \\
-\left\langle\mathfrak{f}_{1 u}, \mathfrak{f}_{3}\right\rangle & =-e^{-\omega / 2}\left\langle\mathfrak{f}_{u u}, \mathfrak{f}\right\rangle, & -\left\langle\mathfrak{f}_{2 u}, \mathfrak{f}_{3}\right\rangle & =-e^{-\omega / 2}\left\langle\mathfrak{f}_{v u}, \mathfrak{f}\right\rangle, & -\left\langle\mathfrak{f}_{3 u}, \mathfrak{f}_{3}\right\rangle & =0 .
\end{aligned}
$$

Then it is easy to see that $-\left\langle\mathfrak{f}_{2 u}, \mathfrak{f}_{3}\right\rangle=e^{\omega / 2}$. Further, $\left\langle\mathfrak{f}_{u u}, \mathfrak{f}_{v}\right\rangle$ can be computed by (1.14) as

$$
\left\langle\mathfrak{f}_{u u}, \mathfrak{f}_{v}\right\rangle=\left\langle\mathfrak{f}_{u}, \mathfrak{f}_{v}\right\rangle_{u}-\left\langle\mathfrak{f}_{u}, \mathfrak{f}_{v u}\right\rangle=\omega_{u} e^{\omega}-e^{\omega}\left\langle\mathfrak{f}_{u}, \mathfrak{H}\right\rangle
$$

and thus $\left\langle\mathfrak{f}_{1 u}, \mathfrak{f}_{2}\right\rangle$ can be rephrased as

$$
\left\langle\mathfrak{f}_{1 u}, \mathfrak{f}_{2}\right\rangle=\frac{\omega_{u}}{2}-\left\langle\mathfrak{f}_{u}, \mathfrak{H}\right\rangle=\frac{\omega_{u}}{2}+\ell .
$$

Here, since $\mathfrak{H}$ is the mean curvature vector $\mathfrak{f}$, that is, $\mathfrak{H}$ can be represented by $\left\{\mathfrak{E}_{1}, \mathfrak{E}_{2}\right\}=$ $\left\{\sqrt{-1} e^{-\omega / 2} \mathfrak{f}_{u}, \sqrt{-1} e^{-\omega / 2} \mathfrak{f}_{v}\right\}, \operatorname{Re}\left\langle\mathfrak{H}, \mathfrak{f}_{u}\right\rangle=\operatorname{Re}\left\langle\mathfrak{H}, \mathfrak{f}_{v}\right\rangle=0$ and thus $-\left\langle\mathfrak{f}_{u}, \mathfrak{H}\right\rangle=\left\langle\mathfrak{H}, \mathfrak{f}_{u}\right\rangle=\ell$, that is, $\ell$ and $m$ take purely imaginary values. Similarly, we have $\left\langle\mathfrak{f}_{2 u}, \mathfrak{f}_{1}\right\rangle=-\frac{\omega_{u}}{2}+\ell$. Further since $\left\langle\mathfrak{f}_{u}, \mathfrak{f}\right\rangle=0$ and $\left\langle\mathfrak{f}_{u}, \mathfrak{f}_{u}\right\rangle=0$, we have $\left\langle\mathfrak{f}_{u u}, \mathfrak{f}\right\rangle=0$ and thus $-\left\langle\mathfrak{f}_{1_{u}}, \mathfrak{f}_{3}\right\rangle=0$. By using $\left\langle\mathfrak{f}_{u}, \mathfrak{f}_{u}\right\rangle=0$, the second derivative of $\left\langle\mathfrak{f}_{u}, \mathfrak{f}\right\rangle=0$ with respect to $u$ implies $\left\langle\mathfrak{f}_{u u u}, \mathfrak{f}\right\rangle=-\left\langle\mathfrak{f}_{u u}, \mathfrak{f}_{u}\right\rangle$. Moreover, the derivative of $\left\langle\mathfrak{f}_{u}, \mathfrak{f}_{u}\right\rangle=0$ with respect to $u$ implies that $\operatorname{Re}\left\langle\mathfrak{f}_{u u}, \mathfrak{f}_{u}\right\rangle=0$, thus $Q=\left\langle\mathfrak{f}_{\text {uuu }}, \mathfrak{f}\right\rangle$ takes purely imaginary values and $\left\langle\mathfrak{f}_{1 u}, \mathfrak{f}_{1}\right\rangle=-Q e^{-\omega}$.

Finally we obtain $\mathcal{U}$ as in (1.15). A similar computation for $\mathcal{F}^{-1} \mathcal{F}_{v}$ shows that $\mathcal{V}$ is as in (1.16).

Definition 2. By using the purely imaginary functions $Q, R, \ell$ and $m$ in (1.17), we define two differentials as

$$
\begin{aligned}
C & =Q \mathrm{~d} u^{3}+R \mathrm{~d} v^{3}=\left\langle\mathfrak{f}_{u u u}, \mathfrak{f}\right\rangle \mathrm{d} u^{3}+\left\langle\mathfrak{f}_{v v v}, \mathfrak{f}\right\rangle \mathrm{d} v^{3}, \\
L & =\ell \mathrm{d} u+m \mathrm{~d} v=\left\langle\mathfrak{H}, \mathfrak{f}_{u}\right\rangle \mathrm{d} u+\left\langle\mathfrak{H}, \mathfrak{f}_{v}\right\rangle \mathrm{d} v .
\end{aligned}
$$

The form $C$ will be called the cubic differential and $L$ will be called the mean curvature 1-form (some authors also call it "Maslov form"). Both forms take purely imaginary values.

Remark 1.9. The cubic differential $C$ and the mean curvature 1 -form $L$ are defined by using a horizontal lift $\mathfrak{f}$ instead of the original immersion $f$, however, $C$ and $L$ are independent of the choice of a horizontal lift. Thus they are an invariant of the timelike Lagrangian immersion $f$. 
1.3. Fundamental theorem. The Maurer-Cartan form $\alpha=\mathcal{F}^{-1} \mathrm{~d} \mathcal{F}$ of the coordinate frame for a timelike Lagrangian immersion $f$ in $\mathbb{C H}_{1}^{2}$ satisfies the Maurer-Cartan equation $\mathrm{d} \alpha+\alpha \wedge \alpha=0$, that is

$$
\mathcal{U}_{v}-\mathcal{V}_{u}+[\mathcal{V}, \mathcal{U}]=0
$$

holds. Then a straightforward computation shows that we have the following system of partial differential equations:

$$
\begin{aligned}
& \omega_{u v}=e^{\omega}-Q R e^{-2 \omega}+m \ell, \\
& \ell_{v}-m_{u}=0, \\
& Q_{v} e^{-2 \omega}+\left(e^{-\omega} \ell\right)_{u}=0, \quad R_{u} e^{-2 \omega}+\left(e^{-\omega} m\right)_{v}=0 .
\end{aligned}
$$

In the following we show the fundamental theorem of timelike Lagrangian surfaces in $\mathbb{C H}_{1}^{2}$.

Theorem 1.10. Let $f: \mathbb{D} \rightarrow \mathbb{C H}_{1}^{2}$ be a timelike Lagrangian immersion and $\mathfrak{f}$ a horizontal lift of $f$. Further let $\mathrm{d} s^{2}=2 e^{\omega} \mathrm{d} u \mathrm{~d} v, C=Q \mathrm{~d} u^{3}+R \mathrm{~d} v^{3}$ and $L=\ell \mathrm{d} u+m \mathrm{~d} v$ be the metric, the cubic differential and the mean curvature 1-form of $f$. Then these functions $\omega, Q, R, \ell$ and $m$ satisfy the system of partial differential equations (1.21), (1.22) and (1.23).

Conversely let $\mathrm{d} s^{2}=2 e^{\omega} \mathrm{d} u \mathrm{~d} v, C=Q \mathrm{~d} u^{3}+R \mathrm{~d} v^{3}$ and $L=\ell \mathrm{d} u+m \mathrm{~d} v$ be defined by solutions of the system of partial differential equations (1.21), (1.22) and (1.23) with purely imaginary $Q, R, \ell$ and $m$. Then there exists a timelike Lagrangian immersion $f$ such that the metric, the cubic differential and the mean curvature 1-form are $\mathrm{d} s^{2}, C$ and $L$, respectively.

Proof. We only need to prove the converse. Since $\omega, C$ and $L$ satisfy (1.21), (1.22) and (1.23), there exists an $\mathcal{F}: \mathbb{D} \rightarrow \mathrm{U}_{2,1}$ such that $\mathcal{F}^{-1} \mathrm{~d} \mathcal{F}=\mathcal{U} \mathrm{d} u+\mathcal{V} \mathrm{d} v$ with $\mathcal{U}$ and $\mathcal{V}$ defined in (1.15) and (1.16). Let $e_{3}=(0,0,1)^{T}$ and set $\mathfrak{f}=\mathcal{F} e_{3}$. Then it is easy to see that $\mathfrak{f}$ takes values in $H_{3}^{5}:\langle\mathfrak{f}, \mathfrak{f}\rangle=\left\langle\mathcal{F} e_{3}, \mathcal{F} e_{3}\right\rangle=-|a|^{2}$, if $\mathcal{F}=a \mathcal{F}_{0}$, where the latter matrix has determinant 1. But the determinant of $\mathcal{F}$ is in $S^{1}$, whence $|a|=1$. Moreover, it is also straightforward to see

$$
\left\langle\mathfrak{f}_{u}, \mathfrak{f}_{u}\right\rangle=\left\langle\mathfrak{f}_{v}, \mathfrak{f}_{v}\right\rangle=0
$$

and $\left\langle\mathfrak{f}_{u}, \mathfrak{f}_{v}\right\rangle$ takes values in $\mathbb{R}^{\times}$, that is, $\mathfrak{f}$ is timelike, is parametrized by null coordinates and is Legendrian, that is, $\operatorname{Im}\left\langle\mathfrak{f}_{u}, \mathfrak{f}_{v}\right\rangle=0$ holds. Finally taking the Boothby-Wang fibration $\pi: H_{3}^{5} \rightarrow \mathbb{C H}_{1}^{2}$ for $\mathfrak{f}$, that is $f=\pi \circ \mathfrak{f}$, we have a timelike Lagrangian immersion $f$ in $\mathbb{C H}_{1}^{2}$.

1.4. Minimality. In the following, we characterize minimality of a timelike Lagrangian immersion in $\mathbb{C H}_{1}^{2}$ in terms of the invariant 1 -form $L$ defined in the previous section.

Proposition 1.11. Let $f: \mathbb{D} \rightarrow \mathbb{C H}_{1}^{2}$ be a timelike Lagrangian immersion and $L$ the differential 1 -form defined in (1.20). Then $L$ is closed. Moreover, let $\mathcal{L}$ denote a purely imaginary integral of $L$ on $\mathbb{D}$. Then the diagonal matrix

$$
D=\exp [\operatorname{diag}(-\mathcal{L},-\mathcal{L}, 0)]=\operatorname{diag}(\exp [-\mathcal{L},], \exp [-\mathcal{L},], 1)
$$

is well-defined and $\operatorname{det}(\mathcal{F} D)$ is constant. Furthermore, $f$ is minimal if and only if $L \equiv 0$ if and only if the determinant $\operatorname{det} \mathcal{F}$ is constant. Thus, without loss of generality we can assume that $\operatorname{det} \mathcal{F} \equiv 1$ holds.

Proof. The closedness of $L$ follows from (1.22). Thus

$$
D=\operatorname{diag}(\exp [-\mathcal{L}], \exp [-\mathcal{L}], 1)
$$


is well-defined. It is also easy to see that $\operatorname{Tr}\left\{(\mathcal{F} D)^{-1} \mathrm{~d}(\mathcal{F} D)\right\}=0$, thus $\operatorname{det}(\mathcal{F} D)$ is constant. Since $\mathcal{L}$ is only determined up to a purely imaginary constant, we can adjust this constant such that the determinant of $\mathcal{F} D$ is identically 1 .

Moreover, by (1.20) the mean curvature vector $\mathfrak{H}$ vanishes if and only if $\ell=m=0$ and this is equivalent with $L=0$.

Remark 1.12 .

(1) Since $L$ takes purely imaginary values, the function $\exp (2 \mathcal{L})$ takes values $S^{1}$. It is called Lagrangian angle function of $f$, [21, after Lemma 3.1].

(2) Note that $\mathcal{F} D$ is in $\mathrm{U}_{2,1}$. The last column of this matrix is the same (horizontal lift) as the one of $\mathcal{F}$. However, the first two columns are rotated. Hence $\mathcal{F} D$ is in general no longer a coordinate frame of some timelike Lagrangian immersion.

(3) Since $\mathcal{F} D$ also has constant determinant (in $S^{1}$ ), one can easily change to a new "frame" which has determinant 1 . This can be done naturally in several ways: one can multiply the matrix $\mathcal{F} D$ by $a^{-1 / 3}$, where $a=\operatorname{det}(\mathcal{F} D)$ is or one can multiply on the right by the matrix $\hat{D}=\operatorname{diag}\left(a^{-1 / 2}, a^{-1 / 2}, 1\right)$. Actually, by replacing the lift $\mathfrak{f}$ of the original timelike Lagrangian immersion $f$ by the lift $\hat{\mathfrak{f}}=a^{-1} \mathfrak{f}, a \in S^{1}$, one obtains the same mean curvature 1 -form $L$ and thus automatically $\hat{\mathcal{F}} D(0,0)=\mathrm{id}$, whence also $\operatorname{det}(\mathcal{F} D) \equiv 1$.

(4) If a timelike Lagrangian surface $f$ is minimal, then of the two normalizations just discussed the last option seems to be preferable, since in this case the new immersion $\hat{\mathfrak{f}}$ leads directly to a coordinate frame which satisfies $\mathcal{F}(0,0)=\mathrm{id}$.

(5) It is known that there are no compact minimal surfaces in a pseudo-Riemannian manifold with non-positive curvature, see for example [19, p. 379].

\section{Characterization of a timelike minimal Lagrangian surfaces}

In this section, we characterize a timelike minimal Lagrangian surface in terms of a family of flat connections. For this purpose, we first consider the associated family of a timelike minimal Lagrangian surface and naturally introduce the so-called "spectral parameter" $\lambda$ into the Maurer-Cartan form $\alpha$ of the coordinate frame $\mathcal{F}$. In Theorem 2.3, we characterize the minimality of a timelike Lagrangian surface in terms of a family of connections $\mathrm{d}+\alpha^{\lambda}$.

2.1. Associated family. Let $f: M \rightarrow \mathbb{C H}_{1}^{2}$ be a timelike minimal Lagrangian immersion. Then there exists the metric $2 e^{\omega} \mathrm{d} u \mathrm{~d} v$, the cubic differential $C$ and the mean curvature 1form $L$ which vanishes identically on $M$ associated to $f$. Then it is clear from $(1.21),(1.22)$ and (1.23), that the integrability conditions for a minimal surface are $m=\ell=0$ and the partial differential equation

$$
\omega_{u v}=e^{\omega}-Q R e^{-2 \omega},
$$

with purely imaginary functions $Q$ and $R$ satisfying $Q_{v}=0$ and $R_{u}=0$, and a real valued function $\omega$.

Remark 2.1. The equation (2.1) is the original Tzitzéica equation of indefinite affine spheres up to sign which can be easily adjusted by the change of coordinates $(u, v)$. However, the affine spheres have real cubic differential and the timelike minimal Lagrangian surfaces in 
$\mathbb{C H}_{1}^{2}$ have purely imaginary cubic differential, thus a solution of the Tzitzéica equation gives two different classes of surfaces.

In this case, by Theorem 1.10 , there exists a family of solutions parametrized by $\lambda \in \mathbb{R}_{>0}$

$$
\left\{e^{\omega^{\lambda}}, C^{\lambda}, L^{\lambda}\right\}_{\lambda \in \mathbb{R}_{>0}}
$$

such that

$$
\omega^{\lambda}=\omega, \quad C^{\lambda}=\lambda^{-3} Q \mathrm{~d} u^{3}+\lambda^{3} R \mathrm{~d} v^{3}, \quad L^{\lambda}=L=0 .
$$

Then by Theorem 1.10, there exists a family of timelike Lagrangian minimal surfaces $\left\{\hat{f}^{\lambda}\right\}_{\lambda \in \mathbb{R}>0}$ such that $\left.\hat{f}^{\lambda}\right|_{\lambda=1}=f$. It is natural to call the family $\left\{\hat{f}^{\lambda}\right\}_{\lambda \in \mathbb{R}_{>0}}$ the associated family of $f$. The parameter $\lambda$ will be called the spectral parameter.

Remark 2.2. It is important to note that the parameter $\lambda$ above can actually be chosen from $\mathbb{C}^{\times}$without restricting the integrability condition. The solutions to some PDE's mentioned in the proof of Theorem 1.10 can thus be computed for all $\lambda \in \mathbb{C}^{\times}$. This is an important information, since in the discussion of the construction method via loop groups one will carry out the group splittings on the unit circle, while one discusses surfaces only for $\lambda \in \mathbb{R}_{>0}$.

Let $\hat{\mathcal{F}}$ be the coordinate frame of a horizontal lift $\hat{\mathfrak{f}}^{\lambda}$ of $\hat{f}^{\lambda}$. Then the Maurer-Cartan form $\hat{\alpha}=\hat{\mathcal{U}} \mathrm{d} u+\hat{\mathcal{V}} \mathrm{d} v$ of $\hat{\mathcal{F}}$ for the associated family $\left\{\hat{f}^{\lambda}\right\}_{\lambda \in \mathbb{R}_{>0}}$ is given by $\hat{\mathcal{U}}$ and $\hat{\mathcal{V}}$ as in (1.15) and (1.16) where we have replaced $Q, R, \ell$ and $m$ by $\lambda^{-3} Q, \lambda^{3} R, 0$ and 0 , respectively.

Then consider

$$
F=\hat{\mathcal{F}} G, \quad G=\operatorname{diag}\left(\lambda, \lambda^{-1}, 1\right)
$$

and thus

$$
\alpha=F^{-1} \mathrm{~d} F=U \mathrm{~d} u+V \mathrm{~d} v
$$

with $U=G^{-1} \hat{\mathcal{U}} G$ and $V=G^{-1} \hat{\mathcal{V}} G$. Since $G$ takes values in $\mathrm{U}_{2,1}$ for any $\lambda \in \mathbb{R}_{>0}$, thus $G \hat{\mathcal{F}} e_{3}$ is isometric to $\hat{\mathcal{F}} e_{3}$. Define $f^{\lambda}=\pi \circ G \hat{\mathcal{F}} e_{3}$. Thus we do not distinguish $\left\{\hat{f}^{\lambda}\right\}_{\lambda \in \mathbb{R}_{>0}}$ and $\left\{f^{\lambda}\right\}_{\lambda \in \mathbb{R}>0}$, and it will be also called the associated family.

2.2. A family of flat connections. Let us return now to the general case of a timelike Lagrangian immersion $f$, with horizontal lift $\mathfrak{f}$ and coordinate frame $\mathcal{F}$ with $D$ defined in (1.24) such that $\mathcal{F} D(0,0)=$ id. Then it is easy to see that the Maurer-Cartan form $(\mathcal{F} D)^{-1} \mathrm{~d}(\mathcal{F} D)=\hat{\mathcal{U}} \mathrm{d} u+\hat{\mathcal{V}} \mathrm{d} v$ can be computed as

$$
\hat{\mathcal{U}}=\left(\begin{array}{ccc}
\frac{\omega_{u}}{2} & m & e^{\omega / 2+\mathcal{L}} \\
-Q e^{-\omega} & -\frac{\omega_{u}}{2} & 0 \\
0 & e^{\omega / 2-\mathcal{L}} & 0
\end{array}\right), \quad \hat{\mathcal{V}}=\left(\begin{array}{ccc}
-\frac{\omega_{v}}{2} & -R e^{-\omega} & 0 \\
\ell & \frac{\omega_{v}}{2} & e^{\omega / 2+\mathcal{L}} \\
e^{\omega / 2-\mathcal{L}} & 0 & 0
\end{array}\right)
$$

From the discussion in the previous section, it is natural to introduce a family of MaurerCartan forms $\alpha^{\lambda}$ for the Maurer-Cartan form $\alpha$ of the timelike Lagrangian surface $f: M \rightarrow$ $\mathbb{C H}_{1}^{2}$ as

$$
\alpha^{\lambda}=U^{\lambda} \mathrm{d} u+V^{\lambda} \mathrm{d} v
$$

for $\lambda \in \mathbb{C}^{\times}$, where $U^{\lambda}$ and $V^{\lambda}$ are given by 


$$
U^{\lambda}=\left(\begin{array}{ccc}
\frac{\omega_{u}}{2} & \lambda m & \lambda^{-1} e^{\omega / 2+\mathcal{L}} \\
-\lambda^{-1} Q e^{-\omega} & -\frac{\omega_{u}}{2} & 0 \\
0 & \lambda^{-1} e^{\omega / 2-\mathcal{L}} & 0
\end{array}\right), V^{\lambda}=\left(\begin{array}{ccc}
-\frac{\omega_{v}}{2} & -\lambda R e^{-\omega} & 0 \\
\lambda^{-1} \ell & \frac{\omega_{v}}{2} & \lambda e^{\omega / 2+\mathcal{L}} \\
\lambda e^{\omega / 2-\mathcal{L}} & 0 & 0
\end{array}\right) .
$$

Note that in this general situation we permit, opposite to the last subsection, $m \neq 0$ and $\ell \neq 0$.

It is clear that $\left.\alpha^{\lambda}\right|_{\lambda=1}$ is the Maurer-Cartan form of the frame $\mathcal{F} D$ of $f$. In the following theorem using the family of Maurer-Cartan forms $\alpha^{\lambda}$, we characterize, when a timelike Lagrangian surface in $\mathbb{C H}_{1}^{2}$ actually is minimal.

Theorem 2.3. Let $f: \mathbb{D} \rightarrow \mathbb{C H}_{1}^{2}$ be a timelike Lagrangian surface in $\mathbb{C H}_{1}^{2}$. Then the following statements are equivalent:

(1) $f$ is minimal.

(2) The mean curvature 1 -form $L=\ell \mathrm{d} u+m \mathrm{~d} v$ vanishes.

(3) $\mathrm{d}+\alpha^{\lambda}$ gives a family of flat connections on $\mathbb{D} \times \mathrm{U}_{2,1}$.

Moreover, if any of these three statements above holds, then we have $Q_{v}=0$ and $R_{u}=0$.

Proof. The equivalence $(1) \Leftrightarrow(2)$ follows from Proposition 1.11. Let us compute the flatness of $\mathrm{d}+\alpha^{\lambda}$. In terms of $U^{\lambda}$ and $V^{\lambda}$, it is equivalent with $U_{v}^{\lambda}-V_{u}^{\lambda}+\left[V^{\lambda}, U^{\lambda}\right]=0$, and a straightforward computation shows that this is equivalent with the following equations:

$$
\begin{aligned}
& \omega_{u v}-e^{\omega}+Q R e^{-2 \omega}-m \ell=0, \\
& \left(m e^{-\omega}\right)_{v}+R_{u} e^{-2 \omega}=0, \quad\left(\ell e^{-\omega}\right)_{u}+Q_{v} e^{-2 \omega}=0, \\
& \left(\lambda^{-1}-\lambda^{2}\right) m=0, \quad\left(\lambda^{-2}-\lambda\right) \ell=0 .
\end{aligned}
$$

The first three equations are just (1.21) and (1.23), respectively. The remaining two equations are satisfied for all $\lambda \in \mathbb{C}^{\times}$if and only if $m=\ell=0$ and this is equivalent with that $f$ is minimal. This completes the proof.

Remark 2.4. The choices of $U^{\lambda}$ and $V^{\lambda}$ in (2.8) are natural in view of the quasi 6-symmetric space (induced by the order 6 automorphism $\hat{\sigma}$ ) in Section 5. The Maurer-Cartan form $\alpha$ can be decomposed into the eigenspaces of the order 6 automorphism $\hat{\sigma}$, and the $j$-th degree of the spectral parameter $\lambda$ in the Maurer-Cartan form corresponds to the $j$-th eigenspace. This will be explained in more detail in Section 5.4.

We have thus found by two different approaches to the same restricted matrices depending on $\lambda$ and it is clear that for the minimal timelike Lagrangian case it suffices to consider matrices of the type

$$
\alpha^{\lambda}=U^{\lambda} \mathrm{d} u+V^{\lambda} \mathrm{d} v,
$$

with

$$
U^{\lambda}=\left(\begin{array}{ccc}
\frac{\omega_{u}}{2} & 0 & \lambda^{-1} e^{\omega / 2} \\
-\lambda^{-1} Q e^{-\omega} & -\frac{\omega_{u}}{2} & 0 \\
0 & \lambda^{-1} e^{\omega / 2} & 0
\end{array}\right), \quad V^{\lambda}=\left(\begin{array}{ccc}
-\frac{\omega_{v}}{2} & -\lambda R e^{-\omega} & 0 \\
0 & \frac{\omega_{v}}{2} & \lambda e^{\omega / 2} \\
\lambda e^{\omega / 2} & 0 & 0
\end{array}\right)
$$


and $Q_{v}=0, R_{u}=0$ and $\omega_{u v}-e^{\omega}+Q R e^{-2 \omega}=0$.

Corollary 2.5. Let $Q, R$ be purely imaginary functions and $\omega$ be a real function. Moreover define $U^{\lambda}$ and $V^{\lambda}$ as in (2.8) and $\alpha^{\lambda}=U^{\lambda} \mathrm{d} u+V^{\lambda} \mathrm{d} v$, and assume $\mathrm{d} \alpha^{\lambda}+\alpha^{\lambda} \wedge \alpha^{\lambda}=0$ for $\lambda \in \mathbb{C}^{\times}$. Then $Q_{v}=0, R_{u}=0$ and $\omega_{u v}-e^{\omega}+Q R e^{-2 \omega}=0$, and there exists $a$ family of timelike minimal Lagrangian surfaces $\left\{f^{\lambda}\right\}_{\lambda \in \mathbb{R}^{\times}}$in $\mathbb{C} \mathbb{H}_{1}^{2}$ with the cubic differential $C=\lambda^{-3} Q \mathrm{~d} u+\lambda^{3} R \mathrm{~d} v$ and the induced metric $\mathrm{d} s^{2}=2 e^{\omega} \mathrm{d} u \mathrm{~d} v$.

Definition 3. The solution of $\left(F^{\lambda}\right)^{-1} \mathrm{~d} F^{\lambda}=\alpha^{\lambda}$ defined in (2.7) with $U^{\lambda}$ and $V^{\lambda}$ as in (2.8) and with initial condition $F^{\lambda}(0,0)=$ id will be called the extended frame of a timelike minimal Lagrangian surface $f$. The associated family $\left\{f^{\lambda}\right\}_{\lambda \in \mathbb{R}^{\times}}$is defined by $\left.F^{\lambda} e_{3}\right|_{\lambda \in \mathbb{R}^{\times}}$with $e_{3}=(0,0,1)^{T}$.

Example 1 (Real projective space). Let $Q=R=0$, and $\omega=2 \log \left(\frac{2}{-2+u v}\right)$. Then it is easy to see that $\omega$ is a solution of the Tzitzeica equation $\omega_{u v}=e^{\omega}-Q R e^{-2 \omega}=e^{\omega}$. Then the extended frame $F^{\lambda}$ can be explicitly obtained as

$$
F^{\lambda}=\exp \left(\lambda^{-1} u N_{+}\right)\left(\begin{array}{ccc}
e^{-\omega / 2} & 0 & 0 \\
0 & e^{\omega / 2} & 0 \\
0 & 0 & 1
\end{array}\right) \exp \left(\lambda v N_{-}\right), \quad N_{+}=\left(\begin{array}{lll}
0 & 0 & 1 \\
0 & 0 & 0 \\
0 & 1 & 0
\end{array}\right), \quad N_{-}=-N_{+}^{T} .
$$

Then the horizontal lift $\mathfrak{f}=\left.\mathfrak{f}^{\lambda}\right|_{\lambda=1}$ can be computed explicitly as

$$
\mathfrak{f}=\frac{1}{2-u v}\left(\begin{array}{c}
2 u \\
2 v \\
2+u v
\end{array}\right)
$$

Clearly $\mathfrak{f}$ is the anti-de Sitter sphere $H_{1}^{2}$ in $\mathbb{R}_{2}^{3}$. And the immersion $f=\pi \circ \mathfrak{f}$ is a part of the indefinite real projective space in $\mathbb{C H}_{1}^{2}$.

Example 2 (Clifford type cylinder). Let $Q=-R=\sqrt{-1}$, then $\omega=0$ is a solution of the Tzitzeica equation $\omega_{u v}=e^{\omega}-Q R e^{-2 \omega}$. Then the coefficient matrices of the Maurer-Cartan form of $\alpha=U_{v a c}^{\lambda} \mathrm{d} u+V_{v a c}^{\lambda} \mathrm{d} v$ are constant and the equation $\left(F^{\lambda}\right)^{-1} \mathrm{~d} F^{\lambda}=\alpha^{\lambda}$ can be integrated directly. We obtain $F^{\lambda}=\exp \left(U_{v a c}^{\lambda} u+V_{v a c}^{\lambda} v\right)$ with

$$
U_{v a c}^{\lambda}=\lambda^{-1}\left(\begin{array}{ccc}
0 & 0 & 1 \\
-\sqrt{-1} & 0 & 0 \\
0 & 1 & 0
\end{array}\right), \quad V_{v a c}^{\lambda}=\lambda\left(\begin{array}{ccc}
0 & \sqrt{-1} & 0 \\
0 & 0 & 1 \\
1 & 0 & 0
\end{array}\right) .
$$

Clearly $F$ is the extended frame of some timelike minimal Lagrangian immersion and a direct computation shows that the horizontal lift $\mathfrak{f}=\left.\mathfrak{f}^{\lambda}\right|_{\lambda=1}=\left.F e_{3}\right|_{\lambda=1}$ can be computed as

$$
\mathfrak{f}=F_{0} \mathfrak{f}_{0},
$$

where with $\delta=e^{2 \pi \sqrt{-1} / 3}$ we have

$$
\mathfrak{f}_{0}=\frac{1}{\sqrt{3}}\left(\begin{array}{c}
e^{\sqrt{-1}\left(\delta u-\delta^{2} v\right)} \\
-e^{\sqrt{-1}\left(\delta^{2} u-\delta v\right)} \\
e^{\sqrt{-1}(u-v)}
\end{array}\right), \quad \text { and } \quad F_{0}=\frac{1}{\sqrt{3}}\left(\begin{array}{ccc}
-\sqrt{-1} \delta^{2} & \sqrt{-1} \delta & -\sqrt{-1} \\
\sqrt{-1} \delta & -\sqrt{-1} \delta^{2} & \sqrt{-1} \\
1 & -1 & 1
\end{array}\right) \text {. }
$$

Then an another direct computation shows that $F_{0} \in \mathrm{U}_{2,1}$ and $\left\langle\mathfrak{f}_{0}, \mathfrak{f}_{0}\right\rangle=-1$. The timelike surface $\mathfrak{f}_{0}$ is an analogue of the Clifford torus in $\mathbb{C P}^{2}$, see for example [11]. Let us consider the curves $v=-u+a(a \in \mathbb{R})$, where $u$ and $v$ denote on null coordinates. Then

$$
\left.\mathfrak{f}_{0}\right|_{v=-u+a}=\frac{1}{\sqrt{3}}\left(e^{-\sqrt{-1} u-\delta^{2} a},-e^{-\sqrt{-1} u-\delta a}, e^{2 \sqrt{-1} u-a}\right)^{T}
$$


Therefore the surface close up, and $\mathfrak{f}_{0}$ becomes a cylinder.

\section{LEGENDRIAN LIFTS OF GENERAL TIMELIKE LAGRANGIAN IMMERSIONS INTO $\mathbb{C H}_{1}^{2}$}

We have so far only considered timelike minimal Lagrangian immersions from contractible open domains in $\mathbb{R}^{2}$ into $\mathbb{C H}_{1}^{2}$. In the literature, usually immersions are defined on arbitrary Lorentz surfaces (or, more generally, on any real surface of dimension two).

Thus, when considering a timelike Lagrangian immersion $f: M \rightarrow \mathbb{C H}_{1}^{2}$, the question comes up whether there always exists a Legendrian lift $\mathfrak{f}: M \rightarrow H_{3}^{5}$. As a consequence, the question arises, in what sense, if any, a timelike Legendrian immersion is naturally associated with a given timelike Lagrangian immersion.

At one hand, for any contractible open subset $U$ of $M$ such a lift exists, by what was discussed in the beginning of this paper. So the main question is, in what sense a "global" lift exists. In this section we will show that either a given timelike Lagrangian immersion $f: M \rightarrow \mathbb{C H}_{1}^{2}$ already has a global timelike Legendrian lift $\mathfrak{f}: M \rightarrow H_{3}^{5}$, or there exists a threefold cover $\hat{M}$ of $M$, such that the natural lift of $f$ to $\hat{f}: \hat{M}: \rightarrow \mathbb{C H}_{1}^{2}$ has a timelike Legendrian lift $\hat{\mathfrak{f}}: \hat{M} \rightarrow \mathbb{C H}_{1}^{2}$ of $\hat{f}$.

Definition 4. A Lagrangian map $f: M \rightarrow \mathbb{C H}_{1}^{2}$ is called liftable, if there exists some Legendrian map $\mathfrak{f}: M \rightarrow H_{3}^{5}$ such that $f=\pi \circ \mathfrak{f}$.

3.1. The basic transformation formula for horizontal lifts and frames. Now let $M$ be a Lorentz surface and let $f: M \rightarrow \mathbb{C H}_{1}^{2}$ be a Lagrangian immersion. It is known, see for example [27, Section 3.2], that the universal cover of $M$ is diffeomorphic to $\mathbb{R}^{2}$, where the Lorentz metric, however, is not known, in general. For simplicity we will thus assume without loss of generality that the universal cover actually is equal to $\mathbb{R}^{2}$ and denote it by $\mathbb{D}$.

By $\tilde{\pi}: \mathbb{D} \rightarrow M$ we denote the universal covering of $M$. Then we infer that $\mathbb{D}$ is contractible and $\tilde{f}: \mathbb{D} \rightarrow \mathbb{C H}_{1}^{2}, p \mapsto f \circ \tilde{\pi}(p)$, is again a Lagrangian immersion. Moreover, by what was discussed previously, $\tilde{f}$ admits a global horizontal lift $\tilde{\mathfrak{f}}: \mathbb{D} \rightarrow H_{3}^{5}$. Now it is easy to derive the following

Proposition 3.1. We retain the notation and the assumptions just made above. Let $\pi_{1}(M)$ denote the fundamental group of $M$, considered as a group of Deck transformations acting on $\mathbb{D}$. Then for $\gamma \in \pi_{1}(M)$ we obtain:

(1) $\gamma^{*} \tilde{\mathfrak{f}}: \mathbb{D} \rightarrow H_{3}^{5}$ is another global horizontal lift of $\tilde{f}$.

(2) There exists some uniquely determined scalar $\tilde{c}(\gamma) \in S^{1}$ satisfying

$$
\gamma^{* \tilde{\mathfrak{f}}}=\tilde{c}(\gamma) \tilde{\mathfrak{f}}
$$

(3) The map $\tilde{c}: \pi_{1}(M) \rightarrow S^{1}, \gamma \mapsto \tilde{c}(\gamma)$, is a (well defined) homomorphism.

Proof. Since $\gamma \in \pi_{1}(M)$ acts on $\mathbb{D}$ as

$$
\gamma:(u, v) \rightarrow(x, y), \quad x=\gamma^{x}(u), \quad y=\gamma^{y}(v),
$$


for some strictly increasing one variable functions $\gamma^{x}, \gamma^{y}$, see [17], it follows by a straightforward computation that the map $\gamma^{*} \tilde{f}: \mathbb{D} \rightarrow H_{3}^{5}$ is another global horizontal lift of $\tilde{f}$. Therefore $\gamma^{*} \tilde{\mathfrak{f}}=\tilde{c}(\gamma) \tilde{\mathfrak{f}}$ with some uniquely determined scalar $\tilde{c}(\gamma)$ in $S^{1}$. By the uniqueness statement, it follows that $\tilde{c}: \pi_{1}(M) \rightarrow S^{1}$ is a (well defined) homomorphism.

Next we recall from (1.11) the definition of the "natural coordinate frame" for a Lagrangian immersion and apply it to $\tilde{\mathfrak{f}}$ :

$$
\widetilde{\mathcal{F}}=\left(e^{-\omega / 2} \tilde{\mathfrak{f}}_{u}, e^{-\omega / 2} \tilde{\mathfrak{f}}_{v}, \tilde{\mathfrak{f}}\right)
$$

where $(u, v)$ is a null coordinate system on $\mathbb{D}$. We know that $\widetilde{\mathcal{F}}$ takes values in $\mathrm{U}_{2,1}$. Then $|\operatorname{det} \widetilde{\mathcal{F}}|^{2}=1$ and $\widetilde{\mathcal{F}} \in \mathrm{U}_{2,1}=S^{1} \cdot \mathrm{SU}_{2,1}$ follows. By $(3.2)$

$$
e^{\tilde{\omega}} \mathrm{d} u \mathrm{~d} v=\gamma^{*}\left(e^{\tilde{\omega}} \mathrm{d} u \mathrm{~d} v\right)=\left(\gamma_{u}^{x} \gamma_{v}^{y}\right) e^{\gamma^{*} \tilde{\omega}} \mathrm{d} u \mathrm{~d} v
$$

and

$$
\tilde{c}(\gamma) \tilde{\mathfrak{f}}_{u}(u, v)=\left(\gamma^{*} \tilde{\mathfrak{f}}\right)_{u}(u, v)=\tilde{\mathfrak{f}}_{u}\left(\gamma^{x}(u), \gamma^{y}(v)\right) \cdot \gamma_{u}^{x}(u),
$$

the frame defined by $(3.3)$ for $\gamma^{*} \tilde{\mathfrak{f}}$ yields

$$
\gamma^{*} \widetilde{\mathcal{F}}=\tilde{c}(\gamma) \widetilde{\mathcal{F}} k, \quad \text { where } \quad k=\operatorname{diag}\left(\sqrt{\left(\gamma_{u}^{x}\right)^{-1} \gamma_{v}^{y}}, \sqrt{\gamma_{u}^{x}\left(\gamma_{v}^{y}\right)^{-1}}, 1\right) .
$$

Corollary 3.2. If $f$ is minimal Lagrangian, then the homomorphism $\tilde{c}$ satisfies $\tilde{c}(\gamma)^{3}=1$ for all $\gamma \in \pi_{1}(M)$.

Proof. Since det $k=1$ it suffices to note that under our assumptions the determinant of $\operatorname{det} \widetilde{\mathcal{F}}$ is constant.

Theorem 3.3. Let $f: M \rightarrow \mathbb{C H}_{1}^{2}$ be a timelike minimal Lagrangian immersion and let $\tilde{c}$ be the homomorphism defined above. Moreover let $F$ be the family of frames defined in (2.3). Then we obtain for all $\gamma \in \pi_{1}(M)$ :

$$
\begin{aligned}
& \gamma^{*} \widetilde{\mathcal{F}}=\tilde{c}(\gamma) \widetilde{\mathcal{F}} k, \quad \text { where } k=\operatorname{diag}\left(\sqrt{\left(\gamma_{u}^{x}\right)^{-1} \gamma_{v}^{y}}, \sqrt{\gamma_{u}^{x}\left(\gamma_{v}^{y}\right)^{-1}}, 1\right), \\
& \gamma^{*} F=\tilde{c}(\gamma) F k, \quad \text { for } k \text { as above. }
\end{aligned}
$$

Moreover, the homomorphism $\tilde{c}$ can be considered as a homomorphism into the group consisting of three elements: $\tilde{c}: \pi_{1}(M) \rightarrow \mathbb{X}_{3}$, where $\mathbb{X}_{3}=\left\{1, \delta, \delta^{2}\right\}$ with $\delta=e^{2 \pi \sqrt{-1} / 3}$.

3.2. The main theorem about global horizontal lifts. Using the results just obtained we are able now to clarify the relation between timelike minimal Lagrangian surfaces into $\mathbb{C H}_{1}^{2}$ and timelike minimal Legendrian surfaces into $H_{3}^{5}$.

Theorem 3.4. Let $M$ be a Lorentz surface and let $f: M \rightarrow \mathbb{C H}_{1}^{2}$ be a timelike minimal Lagrangian immersion. Then either $f$ admits a global horizontal lift $\mathfrak{f}: M \rightarrow H_{3}^{5}$ or otherwise there exists a Lorentz surface $\hat{M}$ and a threefold covering $\hat{\pi}_{f}: \hat{M} \rightarrow M$ such that $\hat{f}=f \circ \hat{\pi}_{f}$ is a timelike minimal Lagrangian immersion which admits a global horizontal lift $\hat{\mathfrak{f}}: \hat{M} \rightarrow H_{3}^{5}$.

Proof. Considering the homomorphism $\tilde{c}$ discussed in the last subsection we have only two possibilities: 
Case 1: The homomorphism $\tilde{c}$ is trivial: In this case we have $\gamma^{*} \widetilde{\mathcal{F}}=\widetilde{\mathcal{F}}$ and $\widetilde{\mathcal{F}}$ descends to a horizontal lift $\mathfrak{f}: M \rightarrow H_{3}^{5}$ for $f$.

Case 2: The homomorphism $\tilde{c}$ is not trivial: hence the image of $\tilde{c}$ is the group $\mathbb{X}_{3}$. Let $\Gamma$ denote the kernel of $\tilde{c}$ and put $\hat{M}=\Gamma \backslash \mathbb{D}$. Then $\widetilde{\mathcal{F}}$ descends to a horizontal map

$$
\hat{\mathfrak{f}}: \hat{M} \rightarrow H_{3}^{5},
$$

with $\hat{M}=\Gamma \backslash \mathbb{D}$ and $\hat{\mathfrak{f}}$ satisfies

$$
\gamma^{*} \hat{\mathfrak{f}}=\hat{c}(\gamma) \hat{\mathfrak{f}}
$$

where $\hat{c}: \pi_{1}(M) / \Gamma \rightarrow \mathbb{X}_{3} \subset S^{1}$ is the induced homomorphism.

Clearly, $\Gamma$ is a normal subgroup of $\pi_{1}(M)$. Moreover, let $\xi$ denote some element of $\pi_{1}(M)$ satisfying $\tilde{c}(\xi)=\delta=e^{\frac{2 \pi i}{3}}$ and let $\Xi$ denote the subgroup of $\pi_{1}(M)$ generated by $\xi$. Then the first isomorphism theorem for groups tells us

$$
\pi_{1}(M) / \Gamma \cong \mathbb{X}_{3}
$$

and the second isomorphism theorem for groups tells us

$$
\mathbb{X}_{3} \cong \pi_{1}(M) / \Gamma \cong \Xi \Gamma / \Gamma \cong \Xi / \Xi \cap \Gamma \text {. }
$$

As a consequence, the action of the group $\Xi$ on $\hat{M}$ is realized by the group $\mathbb{X}_{3}$. But the image of $\hat{c}$ is in $S^{1}$ and thus is annihilated by the Boothby-Wang type projection. Thus the map

$$
\hat{f}: \hat{M} \rightarrow \mathbb{C H}_{1}^{2}, \quad \text { given by } \hat{f}=\pi \circ \hat{\mathfrak{f}}
$$

is invariant under the action of $\pi_{1}(\hat{M})$ and actually projects to $f$. The claim now follows from the following statements:

(1) $\hat{f}: \hat{M} \rightarrow \mathbb{C H}_{1}^{2}$ is a timelike minimal Lagrangian immersion with global horizontal lift $\hat{\mathfrak{f}}$,

(2) $\hat{M}$ is a threefold cover of $M$.

Corollary 3.5. Let $M$ be any Lorentz surface and $f: M \rightarrow \mathbb{C H}_{1}^{2}$ a timelike minimal Lagrangian immersion. Then either $f$ admits a global horizontal lift (which then is timelike minimal Legendrian) or there exists a threefold cover $\hat{\pi}: \hat{M} \rightarrow M$ such that $\hat{f}: \hat{M} \rightarrow \mathbb{C H}_{1}^{2}$, given by $\hat{f}=\hat{\pi} \circ f$ has a global minimal Legendrian lift to $H_{3}^{5}$.

Corollary 3.6. If the Lorenz surface is contractible, then the notions of a timelike minimal Lagrangian immersion into $\mathbb{C H}_{1}^{2}$ and a timelike minimal Legendrian immersion from $M$ into $H_{3}^{5}$ are equivalent.

Remark 3.7. The theorem just above shows that the relation between minimal Lagrangian surfaces and timelike minimal Legendrian surfaces is a bit delicate. Each timelike minimal Lagrangian surface from $M$ to $\mathbb{C H}_{1}^{2}$ induces a timelike minimal Legendrian surface in $H_{3}^{5}$ either on $M$ itself, or at least on some threefold cover $\hat{M}$.

On the other hand, a timelike Legendrian surface $f: M \rightarrow H_{3}^{5}$, a horizontal map from the Lorentz surface $M$ to $H_{3}^{5}$, induces trivially a timelike Lagrangian surface $f_{0}: M \rightarrow \mathbb{C H}_{1}^{2}$ by projection to $\mathbb{C H}_{1}^{2}$ via the Boothby-Wang type fibration.

The difficulty in the relation between these surface classes is in the (in)coherence of their domains, as expressed in the theorem above. 


\section{REAL FORMS OF $\Lambda \mathrm{SL}_{3} \mathbb{C}_{\sigma}$}

It is clear that the extended frame $F^{\lambda}$ introduced in the previous section takes values in the loop group of $\mathrm{SU}_{2,1}$. In this section, we show that the loop group corresponding to a timelike minimal Lagrangian surface in $\mathbb{C H}_{1}^{2}$ takes values in a particular real form of $\Lambda \mathfrak{s l}_{3} \mathbb{C}_{\sigma}$ (or more generally of the affine Kac-Moody Lie algebra of type $A_{2}^{(2)}$ ).

4.1. Real forms of $\Lambda \mathfrak{s l}_{3} \mathbb{C}_{\sigma}$. This subsection is a brief digression which is intended to help to put this paper into a larger context.

4.1.1. The setting of this paper. A straightforward computation shows that the MaurerCartan form $\alpha^{\lambda}$ in (2.7) of the extended frame $F^{\lambda}$ satisfies the following two equations (where we write $\alpha(\lambda)=\alpha^{\lambda}$ temporarily):

$$
\hat{\sigma}\left(\alpha\left(\epsilon^{-1} \lambda\right)\right)=\alpha(\lambda), \quad \hat{\tau}(\alpha(\bar{\lambda}))=\alpha(\lambda),
$$

where $\epsilon=e^{\pi \sqrt{-1} / 3}$ is the sixth root of unity, $\hat{\sigma}$ is an order 6 linear outer automorphism of $\mathfrak{s l}_{3} \mathbb{C}$ and $\hat{\tau}$ is an anti-linear involution of $\mathfrak{s l}_{3} \mathbb{C}$ defined as follows:

$$
\hat{\sigma}(X)=-\operatorname{Ad}\left(\operatorname{diag}\left(\epsilon^{2}, \epsilon^{4},-1\right) P_{0}\right) X^{T}
$$

and

$$
\hat{\tau}(X)=-\operatorname{Ad}\left(P_{0}\right) \bar{X}^{T}
$$

where $P_{0}$ is defined in (1.1).

More precisely, the $\alpha$ takes values in the following loop algebra:

$$
\Lambda \mathfrak{s l}_{3} \mathbb{C}_{\sigma}^{\tau}=\left\{g: \mathbb{C}^{\times} \rightarrow \mathfrak{s l}_{3} \mathbb{C} \mid \sigma(g(\lambda))=g(\lambda), \quad \tau(g(\lambda))=g(\lambda) \text { and } g \text { is smooth }\right\},
$$

where we defined $\sigma(g)(\lambda)=\hat{\sigma}\left(g\left(\epsilon^{-1} \lambda\right)\right)$ and $\tau(g)(\lambda)=\hat{\tau}(g(\bar{\lambda}))$. Therefore, the extended frame $F$ takes values in the loop group $\Lambda \mathrm{SL}_{3} \mathbb{C}_{\sigma}^{\tau}$ whose Lie algebra is $\Lambda \mathfrak{s l}_{3} \mathbb{C}_{\sigma}^{\tau}$ :

$$
\Lambda \mathrm{SL}_{3} \mathbb{C}_{\sigma}^{\tau}=\left\{g: \mathbb{C}^{\times} \rightarrow \mathrm{SL}_{3} \mathbb{C} \mid \sigma(g(\lambda))=g(\lambda), \quad \tau(g(\lambda))=g(\lambda) \text { and } g \text { is smooth }\right\},
$$

where $\sigma$ is an order 6 automorphism and $\tau$ is an anti-linear involution defined by $\sigma(g)(\lambda)=$ $\hat{\sigma}\left(g\left(\epsilon^{-1} \lambda\right)\right)$ and $\tau(g)(\lambda)=\hat{\tau}(g(\bar{\lambda}))$ with

$$
\begin{aligned}
& \hat{\sigma}(g)=\operatorname{Ad}\left(\operatorname{diag}\left(\epsilon^{2}, \epsilon^{4},-1\right) P_{0}\right)\left(g^{T}\right)^{-1}, \\
& \hat{\tau}(g)=\operatorname{Ad}\left(P_{0}\right)\left(\bar{g}^{T}\right)^{-1} .
\end{aligned}
$$

The order 6 automorphism $\hat{\sigma}$ and the anti-linear involution naturally arise for minimal Lagrangian surfaces as discussed in Section 2.

4.1.2. The case of $A_{2}^{(2)}$. The present paper deals with the Lie group $\mathrm{SL}_{3} \mathbb{C}$ with an outer automorphism $\hat{\sigma}$ and some anti-linear involution $\hat{\tau}$. It is known [16] that up to isomorphisms the Lie algebra and the order 6 automorphism are uniquely determined, see [8, Section 7] for details.

Therefore, in our discussion above we could only change the anti-linear involution $\tau$ on $\Lambda \mathfrak{s l}_{3} \mathbb{C}_{\sigma}$, the so-called the real form involution. Thus we fix the order 6 automorphism $\sigma$ and discuss the classification of real form involutions. 
In fact we have up to inner isomorphisms the following classification of real forms of $\Lambda \mathfrak{s l}_{3} \mathbb{C}_{\sigma}$, or more generally the affine Lie algebra of $A_{2}^{(2)}$.

Theorem 4.1 ([13]). The real form involutions $\tau$ for $\Lambda \mathfrak{s l}_{3} \mathbb{C}_{\sigma}$ are classified (up to isomorphism) as follows:
(1) $\tau(g)(\lambda)=-\overline{g(1 / \bar{\lambda})}^{T}$,
(2) $\tau(g)(\lambda)=\operatorname{Ad}\left(I_{2,1} P_{0}\right) \overline{g(1 / \bar{\lambda})}$,
(3) $\tau(g)(\lambda)=-\operatorname{Ad}\left(I_{2,1}\right) \overline{g(1 / \bar{\lambda})}^{T}$,
(4) $\tau(g)(\lambda)=\overline{g(\bar{\lambda})}$
(5) $\tau(g)(\lambda)=-\operatorname{Ad}\left(P_{0}\right) \overline{g(\bar{\lambda})}^{T}$,

where $I_{2,1}=\operatorname{diag}(1,1,-1)$. The first three are called the almost compact types and the rest are called the almost split types.

Corollary 4.2. The real form involution (5) in Theorem 4.1 is defined by the anti-linear involution $\hat{\tau}$ defined in (4.2).

Remark 4.3.

(a) Each involution in Theorem 4.1 corresponds a particular special class of surfaces:

(1) Minimal Lagrangian surfaces in $\mathbb{C P}^{2}$.

(2) Elliptic or hyperbolic affine spheres in $\mathbb{R}^{3}$.

(3) Minimal Lagrangian surfaces in $\mathbb{C H}^{2}$.

(4) Indefinite affine spheres in $\mathbb{R}^{3}$.

(5) Timelike minimal Lagrangian surfaces in $\mathbb{C H}_{1}^{2}$.

(b) For the first four cases listed above a loop group procedure has already been developed which allows (at least in principle) to construct all the surfaces of the corresponding class. This is a consequence of the fact that these surfaces can be characterized by a certain "Gauss map" to be harmonic. Actually, a harmonic Gauss map has only been established explicitly for minimal Lagrangian surfaces in $\mathbb{C H}^{2}$, that is, in the case (3). In all other cases the existence of a harmonic Gauss map can be concluded, since the Maurer Cartan form of the naturally associated moving frame admits the insertion of a parameter $\lambda \in S^{1}$ in such a way as it is know to correspond to a primitive harmonic map. Below we will modify the construction of (3) in a generalized way so as to fit the purposes of this paper and to permit to prove a Ruh-Vilms type theorem. For the remaining cases a Gauss map will be constructed elsewhere.

(c) Actually, when trying to cover all surface classes falling under the scheme outlined above one also needs to consider what happens if one considers an anti-linear automorphism which is conjugated by an inner automorphism such that the induced anti-linear automorphism of the loop group/loop algebra still commutes with $\hat{\sigma}$, see [8, Section 7]. As a matter of fact, such cases did already occur in the paper [18] and will also occur at least in case (2) above.

\section{Three Quasi 6-SymmetriC SPACES And GaUss maps}

In this section we define for a timelike Lagrangian surface in $\mathbb{C H}_{1}^{2}$ three quasi 6-symmetric spaces (see Definition 5) as well as associated Gauss maps into these spaces. These Gauss 
maps are essentially the same. In Theorem 5.7 we characterize a timelike minimal Lagrangian surface in $\mathbb{C H}_{1}^{2}$ in terms of its Gauss maps, thus proving a Ruh-Vilms type theorem.

5.1. Various bundles. We first introduce three quasi 6 -symmetric spaces of dimension 7 which are bundles over $H_{3}^{5}$. Our approach follows [23] in spirit, but, as a matter of fact, we translate the work of [23] into an "indefinite version" of that paper. We consider altogether three spaces, $F L_{1}, F L_{2}$, and $F L_{3}$. We first choose a natural basis $e_{1}, e_{2}, e_{3}$ of $\mathbb{C}_{2}^{3}$.

(1) $F L_{1}$ : We now consider $\mathbb{C}_{2}^{3}$ as the real 6-dimensional symplectic vector space given by the symplectic form $\Omega=\operatorname{Im}\langle$,$\rangle . Then the family of oriented maximal Lagrangian$ (三 isotropic) subspaces $\mathrm{n}$ of $\mathbb{C}_{2}^{3}$ form a submanifold of the real Grassmannian 3-spaces of $\mathbb{C}_{2}^{3}$, that is, they form the oriented Lagrangian Grassmannian manifold $\mathrm{L}_{\mathrm{Gr}}\left(3, \mathbb{C}_{2}^{3}\right)$. It is known [28] that $\mathrm{L}_{\mathrm{Gr}}\left(3, \mathbb{C}_{2}^{3}\right)$ can be represented as a homogeneous space $\mathrm{U}_{2,1} / \mathrm{SO}_{2,1}$. If the Lagrangian Grassmannian $\mathrm{L}_{\mathrm{Gr}}\left(3, \mathbb{C}_{2}^{3}\right)$ is, in particular, an orbit of $\mathrm{SU}_{2,1}$, it will be called special Lagrangian Grassmannian and it will be denoted by $\operatorname{SL}_{\mathrm{Gr}}\left(3, \mathbb{C}_{2}^{3}\right)$.

Proposition 5.1. $\mathrm{SU}_{2,1}$ acts transitively on $\mathrm{SL}_{\mathrm{Gr}}\left(3, \mathbb{C}_{2}^{3}\right)$, and we obtain

$$
\mathrm{SL}_{\mathrm{Gr}}\left(3, \mathbb{C}_{2}^{3}\right)=\mathrm{SU}_{2,1} / \mathrm{SO}_{2,1} \text {. }
$$

Next we define:

$$
F L_{1}=\left\{(v, V) \mid v \in H_{3}^{5}, v \in V, V \in \mathrm{SL}_{\mathrm{Gr}}\left(3, \mathbb{C}_{2}^{3}\right)\right\}
$$

It is easy to verify that $\mathrm{SU}_{2,1}$ acts on $F L_{1}$. Note that the natural projection from $F L_{1}$ to $\mathbb{C H}_{1}^{2}$ is a pseudo-Riemannian submersion which is equivariant under the natural group actions. Since $H_{3}^{5}=\mathrm{SU}_{2,1} / \mathrm{SU}_{1,1}$, where $\mathrm{SU}_{1,1}$ means $\mathrm{SU}_{1,1} \times\{1\}$, the stabilizer at

$$
\left(e_{3}, \operatorname{span}_{\mathbb{R}}\left\{e_{1}, e_{2}, e_{3}\right\}\right) \in F L_{1}
$$

is clearly given by $\mathrm{SU}_{1,1} \cap \mathrm{SO}_{2,1}$, that is

$$
\mathrm{SO}_{1,1}=\left\{\left(a, a^{-1}, 1\right) \mid a \in \mathbb{R}^{\times}\right\} .
$$

Therefore

$$
F L_{1}=\mathrm{SU}_{2,1} / \mathrm{SO}_{1,1} \text {. }
$$

(2) $F L_{2}$ : For the definition of $F L_{2}$, we consider certain "special regular complex flags" in $\mathbb{C}_{2}^{3}$. Here by a regular complex flag we mean a sequence of four complex subspaces, $Q_{0}=\{0\} \subset Q_{1} \subset Q_{2} \subset Q_{3}=\mathbb{C}_{2}^{3}$ of $\mathbb{C}_{2}^{3}$, where $Q_{j}$ has complex dimension $j$. We then define the notion of a special regular complex flag in $\mathbb{C}_{2}^{3}$ over $q \in H_{3}^{5}$ by requiring that we have a regular complex flag in $\mathbb{C}_{2}^{3}$, where the space $Q_{1}$ satisfies $Q_{1}=\mathbb{C} q$.

Thus we define:

$$
F L_{2}=\left\{(w, W) \mid w \in H_{3}^{5}, w \in W, W \text { is a special regular complex flag in } \mathbb{C}_{2}^{3}\right\} .
$$

The definition of a special flag means that one can find three vectors, $q_{1}, q_{2}, q_{3} \in \mathbb{C}_{2}^{3}$ with $q=q_{3}$, such that (using the signature of $\mathbb{C}_{2}^{3}$ ) the vectors $q_{1}$ and $q_{2}$ span a subspace with signature $(1,1)$. So we obtain a triple $q_{1}, q_{2}$ and $q_{3}$ as discussed in the previous case. By an argument analogous to the previous case we conclude that $\mathrm{SU}_{2,1}$ acts transitively on the family of special flags. Then the stabilizer of the action at a point $\left(e_{3}, 0 \subset \mathbb{C} e_{3} \subset \mathbb{C} e_{3} \oplus \mathbb{C} e_{2} \subset\right.$ $\left.\mathbb{C}_{3} \oplus \mathbb{C} e_{2} \oplus \mathbb{C} e_{1}\right)$ is again given by $\mathrm{SO}_{2,1} \cap$ diag, where diag denotes the set of all diagonal matrices in $\mathrm{SU}_{2,1}$. Thus it is again $\mathrm{SO}_{1,1}$. 
Proposition 5.2. $\mathrm{SU}_{2,1}$ acts transitively on $F L_{2}$, and it can be represented as

$$
F L_{2}=\mathrm{SU}_{2,1} / \mathrm{SO}_{1,1} \text {. }
$$

Note that the natural projection from $F L_{2}$ to $\mathbb{C H}_{1}^{2}$ is a pseudo-Riemannian submersion which is equivariant under the natural group actions.

(3) $F L_{3}$ : Finally, using the isometry group $\mathrm{SU}_{2,1}$ of $H_{3}^{5}$, we can directly define a homogeneous space $F L_{3}$ as

$$
F L_{3}=\left\{U P_{1} U^{T} \mid U \in \mathrm{SU}_{2,1} \text { and } P_{1}=\operatorname{diag}\left(\epsilon^{2}, \epsilon^{4},-1\right) P_{0}\right\},
$$

where $\epsilon=e^{\pi \sqrt{-1} / 3}$ and $P_{0}=\left(\begin{array}{ccc}0 & 1 & 0 \\ 1 & 0 & 0 \\ 0 & 0 & -1\end{array}\right)$ as defined in (1.1).

Theorem 5.3. We retain the assumptions and the notion above. Then the following statements hold:

(1) The spaces $F L_{j},(j=1,2,3)$ are homogeneous under the natural action of $\mathrm{SU}_{2,1}$.

(2) The homogeneous space $F L_{j},(j=1,2,3)$ can be represented as

$$
F L_{j}=\mathrm{SU}_{2,1} / \mathrm{SO}_{1,1}, \quad \text { where } \mathrm{SO}_{1,1}=\left\{\operatorname{diag}\left(a, a^{-1}, 1\right) \mid a \in \mathbb{R}^{\times}\right\} .
$$

In particular they are all 7-dimensional.

Proof. (1), (2): The statements clearly follow from Proposition 5.1, Proposition 5.2 and the definition of $F L_{3}$ in (5.2) and the stabilizer at $P_{1}$ is easily computed as $\mathrm{SO}_{1,1}$.

5.2. Quasi $k$-symmetric spaces. It is easy to prove that the fixed point set of the real form involution $\hat{\tau}$ in (4.6) of $\mathrm{SL}_{3} \mathbb{C}$ is isomorphic to $\mathrm{SU}_{2,1}$, that is,

$$
\mathrm{SU}_{2,1} \cong\left\{g \in \mathrm{SL}_{3} \mathbb{C} \mid \hat{\tau}(g)=g\right\} .
$$

On the one hand, the order 6 automorphism $\hat{\sigma}$ in (4.5) acting on $\mathrm{SL}_{3} \mathbb{C}$ does not naturally act on $\mathrm{SU}_{2,1}$, since $\hat{\sigma}$ and $\hat{\tau}$ do not commute. However they have the following relation

$$
\hat{\sigma} \hat{\tau} \hat{\sigma}=\hat{\tau} .
$$

By abuse of notation we will also denote the order 6 automorphism and the real form involution on $\mathfrak{s l}_{3} \mathbb{C}$ by $\hat{\sigma}$ and $\hat{\tau}$, respectively. Let $x_{j}, j \in\{0,1, \ldots, 5\}$, be an eigenvector of $\hat{\sigma}$ for the eigenvalue $\epsilon^{j}=e^{2 \pi \sqrt{-1} j / 6}$, that is, $\hat{\sigma} x_{j}=\epsilon^{j} x_{j}$. Then (5.3) yields

$$
\hat{\sigma} \hat{\tau}\left(x_{j}\right)=\epsilon^{j} \hat{\tau}\left(x_{j}\right) .
$$

So $\hat{\tau}$ leaves invariant the eigenspaces $\mathfrak{g}_{j}^{\mathbb{C}} \subset \mathfrak{s l}_{3} \mathbb{C}$ of $\hat{\sigma}$. And the fixed point algebra of $\hat{\tau}$ is spanned by all elements of the form $x_{j}+\hat{\tau}\left(x_{j}\right),(j=0,1, \ldots, 5)$ and $x_{j}$ arbitrary in $\mathfrak{g}_{j}^{\mathbb{C}}$. So the real form decomposes according to the eigenspaces of $\hat{\sigma}$ and we have

$$
\hat{\sigma}\left(x_{j}+\hat{\tau}\left(x_{j}\right)\right)=\epsilon^{j}\left(x_{j}+\hat{\tau}\left(x_{j}\right)\right) .
$$

Thus $\hat{\sigma}$ is not an automorphism of the real Lie algebra $\operatorname{Fix}(\hat{\tau})$, but its action on $\operatorname{Fix}(\hat{\tau})$ is easy to describe, see Section 5.4.

Definition 5. Let $G / K$ be a real homogeneous space, and $\hat{\tau}$ a real form involution acting on the complexification $G^{\mathbb{C}}$ of $G$, such that $G=\operatorname{Fix}(\hat{\tau})$. Moreover, let $\hat{\sigma}$ be an order $k(k \geq 2)$ automorphism acting on $G^{\mathbb{C}}$. Then $G / K$ will be called a quasi $k$-symmetric space if the following conditions are satisfied 
(1) $\hat{\sigma} \hat{\tau} \hat{\sigma}=\hat{\tau}$

(2) $\operatorname{Fix}(\hat{\sigma})^{\circ} \subset K^{\mathbb{C}} \subset \operatorname{Fix}(\hat{\sigma})$.

Remark 5.4. If the automorphism $\hat{\sigma}$ of $G^{\mathbb{C}}$ in fact acts on $G$, then the quasi $k$-symmetric space $G / K$ is just a $k$-symmetric space. However, by condition (1), this happens if and only if $k=2$, and thus a quasi $k$-symmetric space $G / K$ is a $k$-symmetric space if and only if it is a (2-)symmetric space.

Corollary 5.5. The homogeneous spaces $F L_{j}(j=1,2,3)$ are quasi 6-symmetric spaces.

Proof. First we note that the group $G=\mathrm{SU}_{2,1}$ has the complexification $G^{\mathbb{C}}=\mathrm{SL}_{3} \mathbb{C}$ and is the fixed point group of the real form involution $\hat{\tau}$ given in (4.2).

We show that $F L_{3}$ is a quasi 6 -symmetric space. First note that the stabilizer

$$
\operatorname{Stab}_{P_{1}}=\left\{X \in \mathrm{SU}_{2,1} \mid X P_{1} X^{T}=P_{1}\right\} .
$$

at the point $P_{1}=\operatorname{diag}\left(\epsilon^{2}, \epsilon^{4},-1\right) P_{0}$ of $F L_{3}$ is $\mathrm{SO}_{1,1}$. It is easy to verify that the order 6-automorphism $\hat{\sigma}$ of $\mathrm{SL}_{3} \mathbb{C}$ given in (4.5) and the real form involution $\hat{\tau}$ in (4.6) satisfy the condition (1) in Definition 5. Moreover, a direct computation shows that the fixed point of $\hat{\sigma}$ in $\mathrm{SL}_{3} \mathbb{C}$ is $\mathrm{SO}_{1,1}^{\mathbb{C}}$. Thus $\mathrm{Stab}_{P_{1}}$ satisfies the condition (2) in Definition 5.

Thus $F L_{3}$ is quasi 6-symmetric space in the sense of Definition 5. Furthermore, for any pair of homogeneous spaces $F L_{j}$ and $F L_{m}$ there exists a diffeomorphism

$$
\phi_{j m}: F L_{m} \rightarrow F L_{j}
$$

and a homomorphism $\chi_{j m}: \mathrm{SU}_{2,1} \rightarrow \mathrm{SU}_{2,1}$ such that for any $g \in \mathrm{SU}_{2,1}$ and $p \in F L_{m}$ we have

$$
\phi_{j m}(g . p)=\chi_{j m}(g) \cdot \phi_{j m}(p),
$$

As a consequence, also $F L_{1}$ and $F L_{2}$ are 6 -symmetric spaces.

5.3. Normalized Gauss maps of timelike Lagrangian surfaces in $\mathbb{C H}_{1}^{2}$. We now define three Gauss maps for a timelike Lagrangian surface $f$ in $\mathbb{C H}_{1}^{2}$. Let us assume that $f$ is defined on a simply connected domain $M$ and that $\mathfrak{f}$ is a horizontal lift of $f$. Then we define the coordinate frame $\mathcal{F}: M \rightarrow \mathrm{U}_{2,1}$ as in (1.11). Moreover, take the diagonal matrix $D$ as in (1.24) and consider the normalized coordinate frame $\mathcal{F} D$. If necessary replacing $\mathfrak{f}$ by $a \mathfrak{f}$ with some constant $a \in S^{1}$, without loss of generality, we can assume

$$
\hat{F}=\mathcal{F} D: M \rightarrow \mathrm{SU}_{2,1},
$$

and $\hat{F}$ will be called the normalized frame. Note that

$$
D=\operatorname{diag}(\exp (-\mathcal{L}), \exp (-\mathcal{L}), 1)
$$

where $\mathcal{L}$ is the integral of the mean curvature 1 -form $L$ as in (1.20). Thus $D$ is well-defined on $M$. Furthermore, the normalized frame is well-defined up to $\mathrm{SO}_{1,1}$, that is, any normalized frame is of the form

$$
\hat{F} k, \quad k \in \mathrm{SO}_{1,1},
$$

since by the freedom of the null coordinates $(u, v)$ by $(s(u), t(v))$, where $s, t$ are positive functions of one variable each. 
Definition 6. Consider the projections $\pi_{j} \circ \hat{F}: M \rightarrow F L_{j}(j=1,2,3)$, where $\pi_{j}: \mathrm{SU}_{2,1} \rightarrow$ $F L_{j}$. Then

$$
g_{j}=\pi_{j} \circ \hat{F} \quad(j=1,2,3)
$$

will be called the normalized Gauss maps of $f$ (with values in $F L_{j}$ ). Note that by (5.6) the maps $g_{j}$ are well-defined on $M$, that is, independent of coordinates.

Our definitions were a priori not very geometric. But by following [23] we find analogously three obvious geometric interpretations of the Gauss map.

For $F L_{1}$ : Let $g_{1}: M \rightarrow F L_{1}$ be given by

$$
p \mapsto\left(\mathfrak{f}(p), \operatorname{span}_{\mathbb{R}}\left\{\left(e^{-\mathcal{L}_{\mathfrak{f}_{u}}}\right)(p),\left(e^{-\mathcal{L}_{\mathfrak{f}_{v}}}\right)(p), \mathfrak{f}(p)\right\}\right),
$$

where $\mathfrak{f}$ is a horizontal lift of $f$ such that $\operatorname{det} \mathcal{F} D=1$.

For $F L_{2}$ : Let $g_{2}: M \rightarrow F L_{2}$ be given by

$$
p \mapsto\left(\mathfrak{f}(p), 0 \subset \mathbb{C} \mathfrak{f}(p) \subset \mathbb{C} \mathfrak{f}(p) \oplus \mathbb{C} \mathfrak{f}_{u}(p) \subset \mathbb{C} \mathfrak{f}(p) \oplus \mathbb{C f}_{u}(p) \oplus \mathbb{C f}_{v}(p)\right) .
$$

On the other hand we can represent the Gauss map $g_{3}$ by using the frame $\hat{F}$ defined in (5.5) as

$$
g_{3}=\hat{F} P_{1} \hat{F}^{T}, \quad \text { with } \quad P_{1}=\operatorname{diag}\left(\epsilon^{2}, \epsilon^{4},-1\right) P_{0},
$$

where $\epsilon=e^{\pi \sqrt{-1} / 3}$ and $P_{0}$ is defined in (1.1).

Remark 5.6.

(1) From the above arguments, it is clear that the normalized Gauss maps $g_{j}$ do not depend on choices of the coordinates $(u, v)$, but only depend on the Lorentz structure of $M$.

(2) It is known that the natural Gauss map $\tilde{g}_{1}$ of a timelike Legendre immersion $\mathfrak{f}: M \rightarrow$ $H_{3}^{5}$ is given by the wedge product of $\mathfrak{f}$ with the tangential Gauss map $\gamma: M \rightarrow$ $\operatorname{Gr}_{\mathbb{R}}\left(2, \mathbb{C}_{2}^{3}\right)$, that is $\tilde{g}_{1}=(\mathfrak{f}, \gamma \wedge \mathfrak{f})$, and that $\tilde{g}_{1}$ takes values in $F L_{1}$ if and only if $\mathfrak{f}$ is minimal (see [23] for example for the $\mathbb{C P}^{2}$ case). Our normalized Gauss map $g_{1}$ takes values in $F L_{1}$ but it does not imply minimality of a timelike Legendre immersion $\mathfrak{f}$ since we rotate the tangential Gauss map $\gamma$ by the factor $e^{-\mathcal{L}} \in S^{1}$, and thus obtain $g_{1}=\left(\mathfrak{f},\left(e^{-\mathcal{L}} \gamma\right) \wedge \mathfrak{f}\right)$

Let $M$ be a two-dimensional Lorentz surface with null coordinates $(u, v) \in \mathbb{D} \subset M$, and let $G / K$ be a quasi $k$-symmetric space, $k>2$ and consider a smooth map $g: M \rightarrow G / K$. Moreover, let $F: \mathbb{D} \rightarrow G$ be a frame of $g$ and $\alpha=F^{-1} \mathrm{~d} F$ be the Maurer-Cartan form of $F$. According to the decomposition of $\mathfrak{g}=\mathfrak{k}+\mathfrak{p}$ of $G / K$ where $\mathfrak{k}$ is the Lie algebra of $K$, we have

$$
\alpha=\alpha_{\mathfrak{k}}+\alpha_{\mathfrak{p}}=\alpha_{\mathfrak{k}}+\alpha_{\mathfrak{p}}^{u}+\alpha_{\mathfrak{p}}^{v}
$$

where the superscripts $u$ and $v$ denote the $u$-and $v$-parts, respectively. Let us denote by $\hat{\sigma}$ also the differential of the order $k$-automorphism, that is, $\hat{\sigma}$ is the order $k$-automorphism of the Lie algebra $\mathfrak{g}^{\mathbb{C}}$ of $G^{\mathbb{C}}$. Then it is easy to see that $\hat{\sigma}$ has the eigenvalues $\left\{\epsilon^{0}, \epsilon^{1}, \ldots, \epsilon^{k-1}\right\}$ with $\epsilon=e^{2 \pi \sqrt{-1} / k}$ and the complexification $\mathfrak{g}^{\mathbb{C}}$ can be decomposed into $k$-eigenspaces as

$$
\mathfrak{g}^{\mathbb{C}}=\mathfrak{g}_{0}^{\mathbb{C}}+\mathfrak{g}_{1}^{\mathbb{C}}+\cdots+\mathfrak{g}_{k-1}^{\mathbb{C}} .
$$

Here $\mathfrak{g}_{j}^{\mathbb{C}}=\left\{X \in \mathfrak{g}^{\mathbb{C}} \mid \hat{\sigma}(X)=\epsilon^{j} X\right\}$. Note that $\mathfrak{g}_{0}^{\mathbb{C}}=\mathfrak{k}^{\mathbb{C}}$. 
Definition 7. We retain the notation as above. A smooth map $g: M \rightarrow G / K$ is called a Lorentz primitive harmonic map if the following conditions hold:

$$
\alpha_{\mathfrak{p}}^{u} \text { and } \alpha_{\mathfrak{p}}^{v} \text { take values in } \mathfrak{g}_{k-1}^{\mathbb{C}} \text { and } \mathfrak{g}_{1}^{\mathbb{C}} \text {, respectively. }
$$

5.4. Characterization of the minimality in terms of the normalized Gauss maps. It is easy to compute the Maurer-Cartan form of the normalized frame $\hat{F}$ as in (5.5), see (2.4):

$$
\hat{\alpha}=\hat{F}^{-1} \mathrm{~d} \hat{F}=\hat{U} \mathrm{~d} u+\hat{V} \mathrm{~d} v
$$

with

$$
\hat{U}=\left(\begin{array}{ccc}
\frac{\omega_{u}}{2} & m & e^{\omega / 2+\mathcal{L}} \\
-Q e^{-\omega} & -\frac{\omega_{u}}{2} & 0 \\
0 & e^{\omega / 2-\mathcal{L}} & 0
\end{array}\right), \quad \hat{V}=\left(\begin{array}{ccc}
-\frac{\omega_{v}}{2} & -R e^{-\omega} & 0 \\
\ell & \frac{\omega_{v}}{2} & e^{\omega / 2+\mathcal{L}} \\
e^{\omega / 2-\mathcal{L}} & 0 & 0
\end{array}\right)
$$

From Section 2.2, is natural to introduce the spectral parameter into the Maurer-Cartan form as follows:

$$
\hat{\alpha}^{\lambda}=\alpha^{\lambda}
$$

where $\alpha^{\lambda}$ is defined in (2.5).

On the other hand a straightforward computation shows that the eigenspaces $\mathfrak{g}_{j}^{\mathbb{C}} \subset \mathfrak{s l}_{3} \mathbb{C}$ of the order 6-automorphism $\hat{\sigma}$ in $(4.1)$ are

$$
\begin{array}{ll}
\mathfrak{g}_{0}^{\mathbb{C}}=\left\{\left(\begin{array}{ccc}
a_{11} & 0 & 0 \\
0 & -a_{11} & 0 \\
0 & 0 & 0
\end{array}\right) \mid a_{11} \in \mathbb{C}\right\}, & \mathfrak{g}_{1}^{\mathbb{C}}=\left\{\left(\begin{array}{ccc}
0 & a_{12} & 0 \\
0 & 0 & a_{23} \\
a_{23} & 0 & 0
\end{array}\right) \mid a_{12}, a_{23} \in \mathbb{C}\right\}, \\
\mathfrak{g}_{2}^{\mathbb{C}}=\left\{\left(\begin{array}{ccc}
0 & 0 & a_{13} \\
0 & 0 & 0 \\
0 & -a_{13} & 0
\end{array}\right) \mid a_{13} \in \mathbb{C}\right\}, & \mathfrak{g}_{3}^{\mathbb{C}}=\left\{\left(\begin{array}{ccc}
a_{11} & 0 & 0 \\
0 & a_{11} & 0 \\
0 & 0 & -2 a_{11}
\end{array}\right) \mid a_{11} \in \mathbb{C}\right\}, \\
\mathfrak{g}_{4}^{\mathbb{C}}=\left\{\left(\begin{array}{ccc}
0 & 0 & 0 \\
0 & 0 & a_{23} \\
-a_{23} & 0 & 0
\end{array}\right) \mid a_{23} \in \mathbb{C}\right\}, & \mathfrak{g}_{5}^{\mathbb{C}}=\left\{\left(\begin{array}{ccc}
0 & 0 & a_{13} \\
a_{21} & 0 & 0 \\
0 & a_{13} & 0
\end{array}\right) \mid a_{21}, a_{13} \in \mathbb{C}\right\} .
\end{array}
$$

We now have the main theorem of this paper.

Theorem 5.7. Let $f: M \rightarrow \mathbb{C H}_{1}^{2}$ be a timelike Lagrangian surface in $\mathbb{C H}_{1}^{2}$. Then the following statements are equivalent:

(1) $f$ is minimal.

(2) The mean curvature 1 -form $L=\ell \mathrm{d} u+m \mathrm{~d} v$ vanishes.

(3) $\mathrm{d}+\hat{\alpha}^{\lambda}$ gives a family of flat connections on $\mathbb{D} \times \mathrm{SU}_{2,1}$.

(4) The normalized Gauss maps $g_{j}(j=1,2,3)$ are respectively Lorentz primitive harmonic maps into the quasi 6-symmetric spaces $F L_{j}(j=1,2,3)$.

Proof. The equivalences of (1), (2) and (3) follow from Theorem 2.3. We now show the equivalence of (4) and (1). First note that the normalized frame $\hat{F}$ is common for all the normalized Gauss maps $g_{1}, g_{2}$ and $g_{3}$. 
From the eigenspace decomposition of $\hat{\sigma}, \alpha_{\mathfrak{p}}^{u}$ and $\alpha_{\mathfrak{p}}^{v}$ can be computed as

$$
\alpha_{\mathfrak{p}}^{u}=\left(\begin{array}{ccc}
0 & m & e^{\omega / 2+\mathcal{L}} \\
-Q e^{-\omega} & 0 & 0 \\
0 & e^{\omega / 2-\mathcal{L}} & 0
\end{array}\right) \mathrm{d} u, \quad \alpha_{\mathfrak{p}}^{v}=\left(\begin{array}{ccc}
0 & -R e^{-\omega} & 0 \\
\ell & 0 & e^{\omega / 2+\mathcal{L}} \\
e^{\omega / 2-\mathcal{L}} & 0 & 0
\end{array}\right) \mathrm{d} v
$$

Thus it is easy to see that $\alpha_{\mathfrak{p}}^{u}$ and $\alpha_{\mathfrak{p}}^{v}$ respectively take values in $\mathfrak{g}_{5}^{\mathbb{C}}$ and $\mathfrak{g}_{1}^{\mathbb{C}}$ if and only if $L=m \mathrm{~d} u+\ell \mathrm{d} v=0$. Therefore by $(5.7), g_{j}(j=1,2,3)$ is a Lorentz primitive harmonic map into $F L_{j}(j=1,2,3)$ if and only if $f$ is minimal.

Acknowledgement: We would like to thank Hui Ma for pointing out to us (5) in Remark 1.12. We would also like to thank the referee for carefully reading the manuscript and for pointing out to us a number of typographical errors in the manuscript.

\section{REFERENCES}

[1] H. Anciaux. Minimal submanifolds in pseudo-Riemannian geometry. World Scientific Publishing Co. Pte. Ltd., Hackensack, NJ, 2011.

[2] P. Baird, J. C. Wood Harmonic morphisms between Riemannian manifolds. London Mathematical Society Monographs. New Series, 29. The Clarendon Press, Oxford University Press, Oxford, 2003.

[3] V. Back-Valente, N. Bardy-Panse, H. Ben Messaoud, G. Rousseau. Formes presque-déployées des algèbres de Kac-Moody: classification et racines relatives. J. Algebra, 171(1):43-96, 1995.

[4] H. Ben Messaoud, G. Rousseau. Classification des formes réelles presque compactes des algèbres de Kac-Moody affines. J. Algebra, 267(2):443-513, 2003.

[5] W. M. Boothby, H. C. Wang. On contact manifolds. Ann. of Math. (2), 68:721-734, 1958.

[6] F. Dillen, L. Vrancken. Lorentzian isotropic Lagrangian immersions. Filomat, 30:10:2857-2867, 2016.

[7] J. Dorfmeister, U. Eitner. Weierstraß-type representation of affine spheres. Abh. Math. Sem. Univ. Hamburg, 71:225-250, 2001.

[8] J. F. Dorfmeister, W. Freyn, S.-P. Kobayashi, E. Wang, Survey on real forms of the complex $A_{2}^{(2)}$-Toda equation. Complex Manifolds, 6:194-227, 2019, arXiv:1902.01558.

[9] J. F. Dorfmeister, J. Inoguchi, S.-P. Kobayashi. Constant mean curvature surfaces in hyperbolic 3-space via loop group. J. Reine Angew. Math., 686:1-36, 2014.

[10] J. Dorfmeister, J. Inoguchi, M. Toda. Weierstraß type representations of timelike surfaces with constant mean curvature, Integrable Systems in Differential Geometry, Contemp. Math. 308:77-99, 2002.

[11] J. F. Dorfmeister, H. Ma. Some new examples of minimal Lagrangian surfaces in $\mathbb{C} P^{2}$. In preparation, 2019.

[12] J. F. Dorfmeister, E. Wang. Definite affine spheres via loop groups I: general theory, In preparation, 2019.

[13] E. Heintze, C. Groß. Finite order automorphisms and real forms of affine Kac-Moody algebras in the smooth and algebraic category. Mem. Amer. Math. Soc. 219:no. 1030, 2012.

[14] J. Inoguchi, M. Toda. Timelike minimal surfaces via loop groups. Acta Appl. Math., 83(3): 313-355, 2004.

[15] J. Inoguchi, S. Udagawa. Affine spheres and finite gap solutions of Tzitzèica equation. J. Phys. Commun., 2(11):115020, 2018.

[16] V. Kac. Infinite-dimensional Lie algebras. Third edition. Cambridge University Press, Cambridge, 1990.

[17] R. S. Kulkarni. An analogue of the Riemann mapping theorem for Lorentz metrics. Proc. Roy. Soc. London Ser. A 401:117-130, 1985.

[18] S.-P. Kobayashi. Real forms of complex surfaces of constant mean curvature. Trans. Amer. Math. Soc., 363(4):1765-1788, 2011.

[19] S. Kobayashi, K. Nomizu, Foundations of differential geometry. Vol. II. Reprint of the 1969 original. A Wiley-Interscience Publication. John Wiley \& Sons, Inc., 1996.

[20] H. Ma, Y. Ma. Totally real minimal tori in $\mathbb{C P}^{2}$. Math. Z., 249(2):241-267, 2005. 
[21] J. Loftin, I. McIntosh. Minimal Lagrangian surfaces in $\mathbb{C} \mathbb{H}^{2}$ and representations of surface groups into SU (2,1). Geom. Dedicata, 162:67-93, 2013.

[22] J. Loftin, I. McIntosh. Cubic differentials in the differential geometry of surfaces. IRMA Lect. Math. Theor. Phys., 27:Handbook of Teichmüller theory. Vol. VI, 231-274, 2016.

[23] I. McIntosh. Special Lagrangian cones in $\mathbb{C}^{3}$ and primitive harmonic maps. J. Lond. Math. Soc. (2) 67(3):769-789, 2003.

[24] H. Reckziegel. Horizontal lifts of isometric immersions into the bundle space of a pseudo-Riemannian submersion. Global Differential Geometry and Global Analysis, Springer Lectures Notes 1156, 264-279, 1984.

[25] A. E. Ruh, J. Vilms. The tension field of the Gauss map. Trans. Amer. Math. Soc. 149:569-573, 1970.

[26] R. A. Shapiro. Pseudo-Hermitian symmetric spaces. Comment. Math. Helv. 46:529-548, 1971.

[27] T. Weinstein. An introduction to Lorentz surfaces, De Gruyter Expositions in Mathematics 22, Walter de Gruyter \& Co., Berlin, 1996.

[28] D. Yuxin On indefinite special Lagrangian submanifolds in indefinite complex Euclidean spaces. $J$. Geom. Phys. 59(6):710-726, 2009.

Fakultät für Mathematik, TU-München, Boltzmann str. 3, D-85747, Garching, Germany

E-mail address: dorfm@ma.tum.de

Department of Mathematics, Hokkaido University, Sapporo, 060-0810, Japan

E-mail address: shimpei@math.sci.hokudai.ac.jp 